\title{
Early and Middle Holocene Human Occupation of the Egyptian Eastern Desert: Sodmein Cave
}

\author{
Pierre M. Vermeersch ${ }^{1}$ - Veerle Linseele ${ }^{1,2}$. \\ Elena Marinova $^{1,2}$ - Wim Van Neer ${ }^{1,2}$. \\ Jan Moeyersons ${ }^{3}$ - Janet Rethemeyer ${ }^{4}$
}

Published online: 10 September 2015

(C) The Author(s) 2015. This article is published with open access at Springerlink.com

\begin{abstract}
In this paper, we discuss human occupation during the Early and Middle Holocene in the Eastern Desert of Egypt, based mainly on the data provided by excavated deposits from the Sodmein Cave, which produced an important Holocene stratigraphic sequence. This sequence is dated by a large number of conventional and AMS ${ }^{14} \mathrm{C}$ dates. It appears that the area was empty of human occupation during the Late Glacial Maximum (LGM) and the earliest Holocene. With improved climatic conditions, humans arrived in the area, as hunter-gatherers using no ceramics, from around 7.1 to $6.4 \mathrm{Ka}$ cal BC. Humans were absent from the cave during the Holocene 8.2 Ka event (ca. 6.3 Ka cal BC). From 6.2 to 5.0 Ka cal BC, herders visited the site on a regular basis importing caprines. The bone evidence for domesticated small stock is very limited at Sodmein but is nevertheless extremely important, as it contains the oldest known specimens for Africa to date. After 5.0 Ka cal BC, the area was almost entirely deserted.
\end{abstract}

Résumé La présente contribution discute de l'occupation humaine au cours de l'Holocène ancien et moyen dans le désert oriental d'Egypte. L'analyse des données fournies par les dépôts de la grotte Sodmein fournit une séquence stratigraphique holocène importante. Cette séquence est datée par un grand nombre de dates

Electronic supplementary material The online version of this article (doi:10.1007/s10437-015-9195-6) contains supplementary material, which is available to authorized users.

Pierre M. Vermeersch

pierre.vermeersch@ees.kuleuven.be

1 Katholieke Universiteit Leuven, Leuven, Belgium

2 Royal Belgian Institute of Natural Sciences, Brussels, Belgium

3 Royal Museum of Central Africa, Tervuren, Belgium

4 University of Cologne, Cologne, Germany 
conventionnelles et AMS ${ }^{14} \mathrm{C}$. Il semble que la région n'ait pas été occupée au cours de la fin du maximum glaciaire (LGM) et au début de l'Holocène. L'arrivée des humains, chasseurs-cueilleurs sans céramique, dans la région, se situe aux environs de 7.1 à 6.4 $\mathrm{Ka}$ calBC au moment de l'amélioration des conditions climatiques. Durant le 'Holocene 8.2 Ka event' ( $6.3 \mathrm{Ka}$ calBC) l'homme semble absent du site. Plus tard, de 6,2 à 5,0 $\mathrm{Ka}$ calBC, des éleveurs, qui ont introduit des caprins, ont visité le site régulièrement. À Sodmein l'évidence osseuse de caprins domestiques, quoique limitée, est néanmoins extrêmement importante, car elle représente à ce jour les plus anciens spécimens connus pour l'Afrique. Après $5.0 \mathrm{Ka}$ calBC la région a été presque entièrement désertée.

Keywords Egypt $\cdot$ Neolithic $\cdot$ Holocene $\cdot$ Herding $\cdot 14 \mathrm{C}$ dating $\cdot$ Prehistory

\section{Introduction}

The Egyptian Western Desert has been surveyed by many research groups, and the data collected provide extensive information for the understanding of the different population groups that visited the area, mainly during the Early and Middle Holocene (Bubenzer et al. 2007). However, understanding of what happened in the Nile Valley during this same time period remains very restricted (Midant-Reynes 2003). Moreover, very little research has been initiated that considers human occupation in the Egyptian Eastern Desert.

The Belgian Middle Egypt Prehistoric Project of Leuven University carried out surveys and excavations in the Egyptian Eastern Desert, mainly along the Red Sea during the winters of 1989, 1993, 1995 and 1999. The project aimed to understand the prehistoric occupation of this area. Surveys resulted in the identification of several sites. These include Middle Stone Age (MSA) sites, such as Sodmein Cave (Prickett 1979, Vermeersch et al. 1996) and Wadi Bili (Vermeersch et al. 2007), Epipalaeolithic sites such as Tree Shelter (Vermeersch 2008) and Sodmein Cave, and also Neolithic sites such as Wadi Bili, El Gouna (Vermeersch et al. 2005b), Tree Shelter, and Sodmein Cave. The present paper discusses data from Sodmein Cave that are relevant to the human occupation of the Egyptian Eastern Desert during the Early and Middle Holocene (Vermeersch 2012). A variety of integrated data from both archaeology and natural sciences (stratigraphy, fauna, plant remains) were collected. They provide us with evidence for a better understanding of the human occupation during the environmental changes of the area. These data will be integrated in an overview of the North African human population dynamics during the Early and Middle Holocene.

The cave (Figs. 1, 2 and 3) is located in the Red Sea Mountains of the Egyptian Eastern Desert, at $26^{\circ} 14^{\prime} 27^{\prime \prime} \mathrm{N}$ and $33^{\circ} 58^{\prime} 12^{\prime \prime} \mathrm{E}$, about $35 \mathrm{~km}$ to the NNW of Quseir in an area where limestone of the Thebes Formation is outcropping. The geology and geomorphology of the Sodmein Cave were studied by Moeyersons et al. (1996, 1999, 2002). The slightly sloping cave floor is $41 \mathrm{~m}$ wide, 10 to $20 \mathrm{~m}$ deep and $18 \mathrm{~m}$ high at the entrance. At the confluence of the Wadi Nakheil and the Wadi El Ambagi, there is a perennial spring. Other springs, now dry, are found at Bir Nakheil and in the Bir el-Anz. 


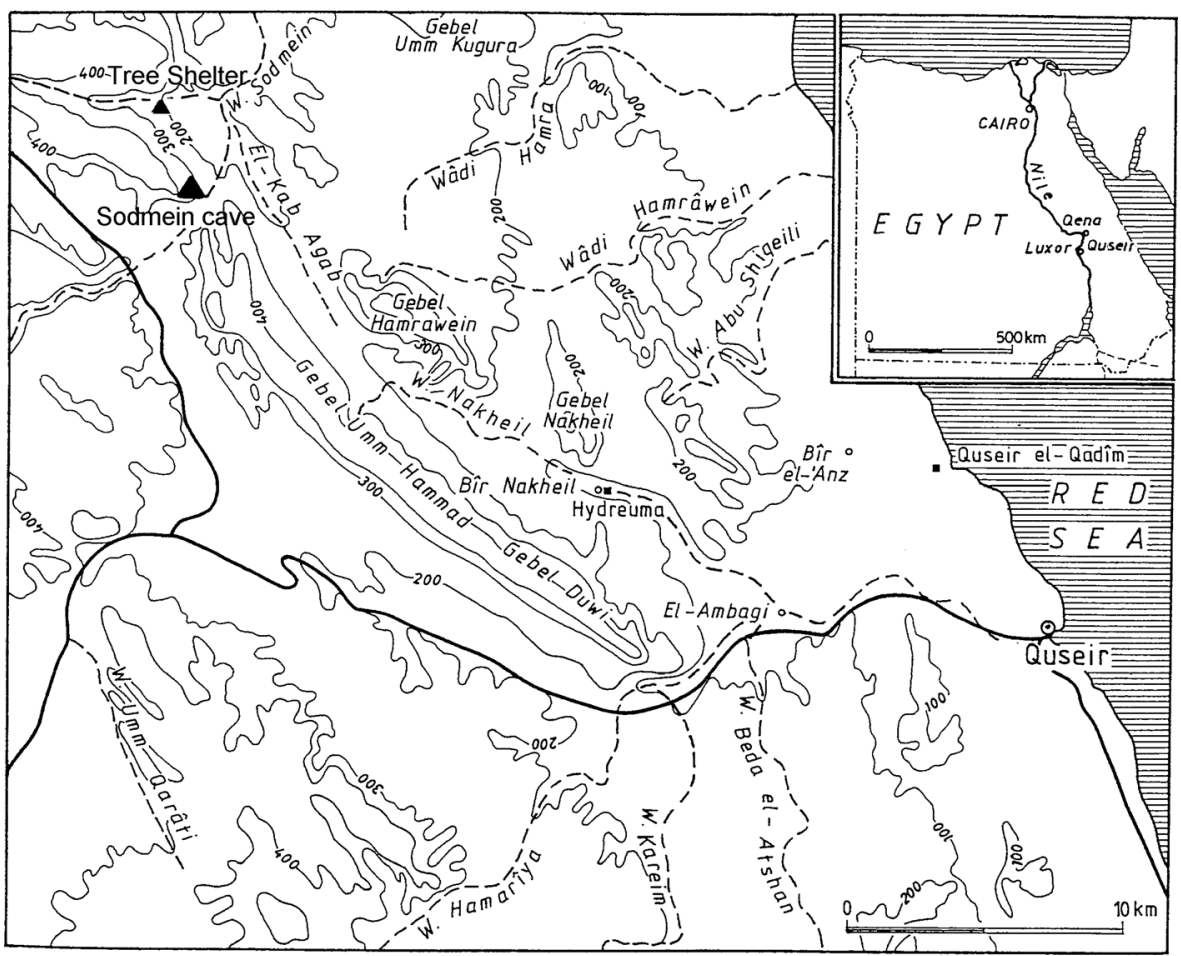

Fig. 1 Topographic map of the area showing the position of the Sodmein Cave and Tree Shelter sites

\section{Field Work}

A general topographical plan was made covering the interior of Sodmein Cave at a contour interval of $20 \mathrm{~cm}$, and the slope outside down into the wadi at a contour interval of $1 \mathrm{~m}$ (Fig. 4). Our excavations proceeded mainly by cutting trenches, laid out

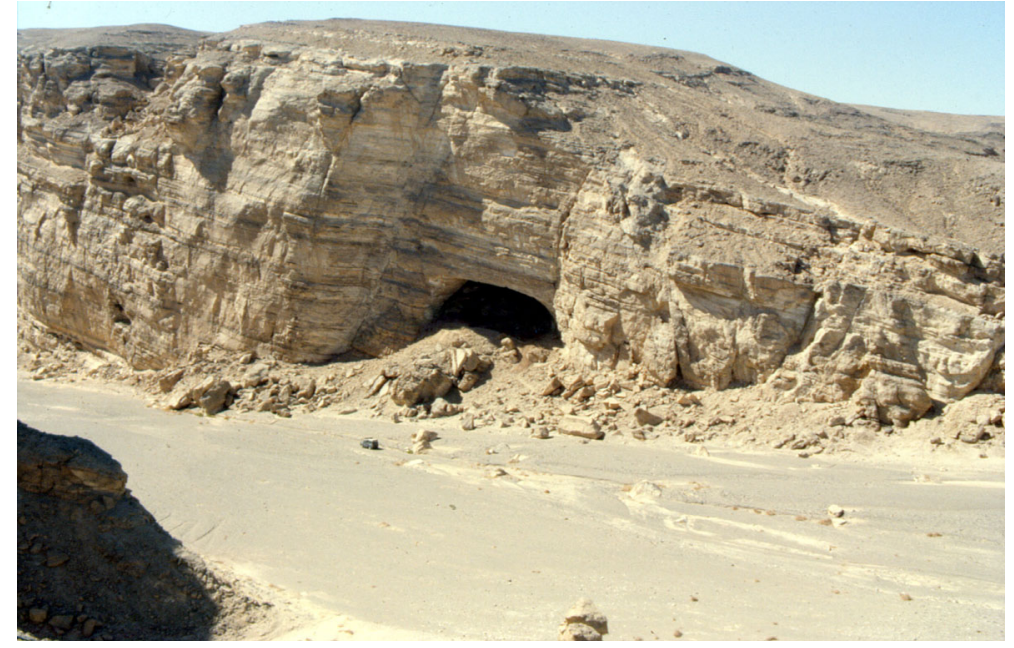

Fig. 2 View to the NW on the cave in the Wadi Sodmein (photo: P. Vermeersch) 


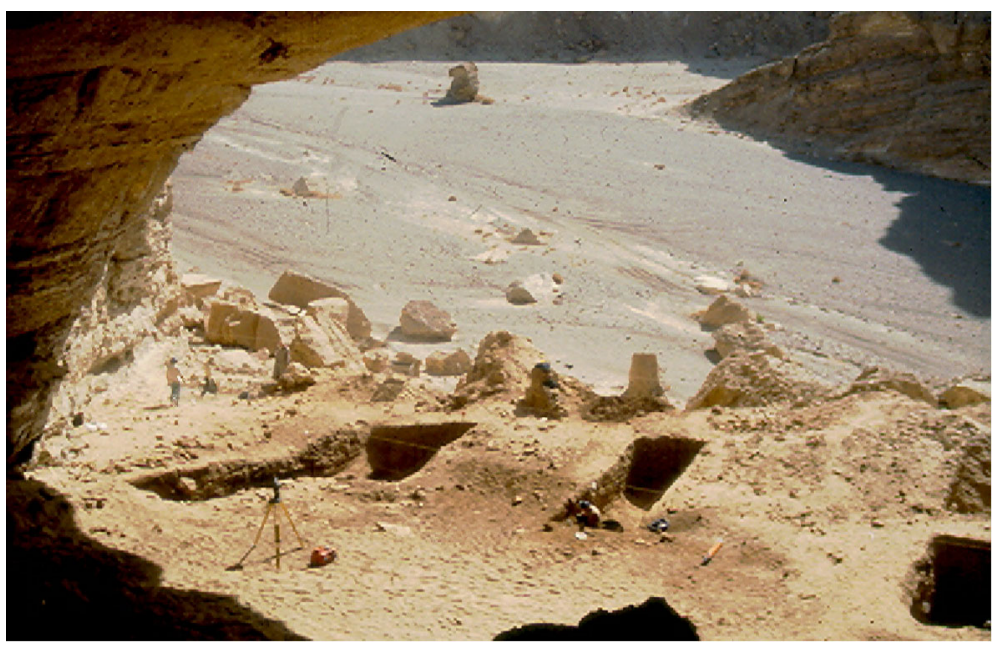

Fig. 3 View from the Sodmein Cave (sector B) over the Wadi Sodmein. (photo: P. Vermeersch)

in different sectors of the site in order to disclose the stratigraphy (additional data are given in Online Resource 1). Screening of deposits on 5-mm mesh was a standard procedure throughout the excavation.

The backwall of the cave and the depositional area in front (Figs. 5 and 6) are covered by a thin veneer of loose loamy sand, containing some angular, 1 - to $15-\mathrm{cm}$

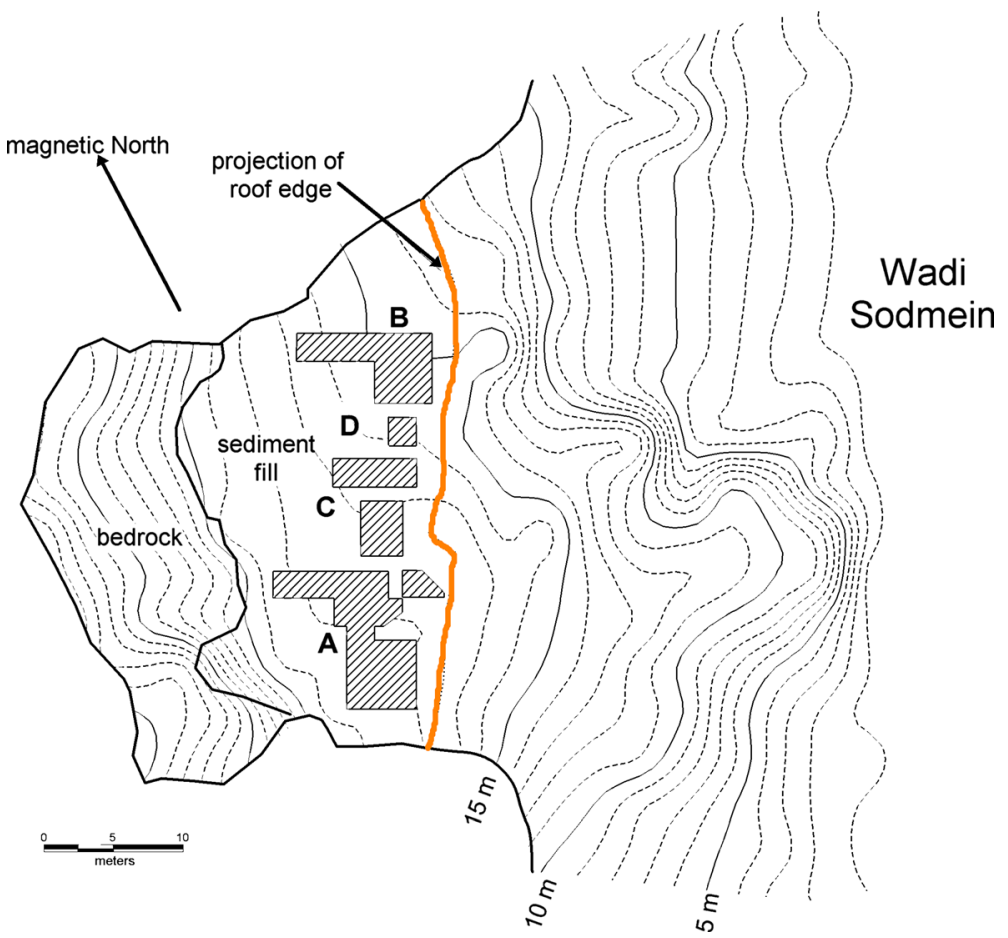

Fig. 4 Topographical map of Sodmein Cave showing the excavation sectors $(A-D)$ and trenches 


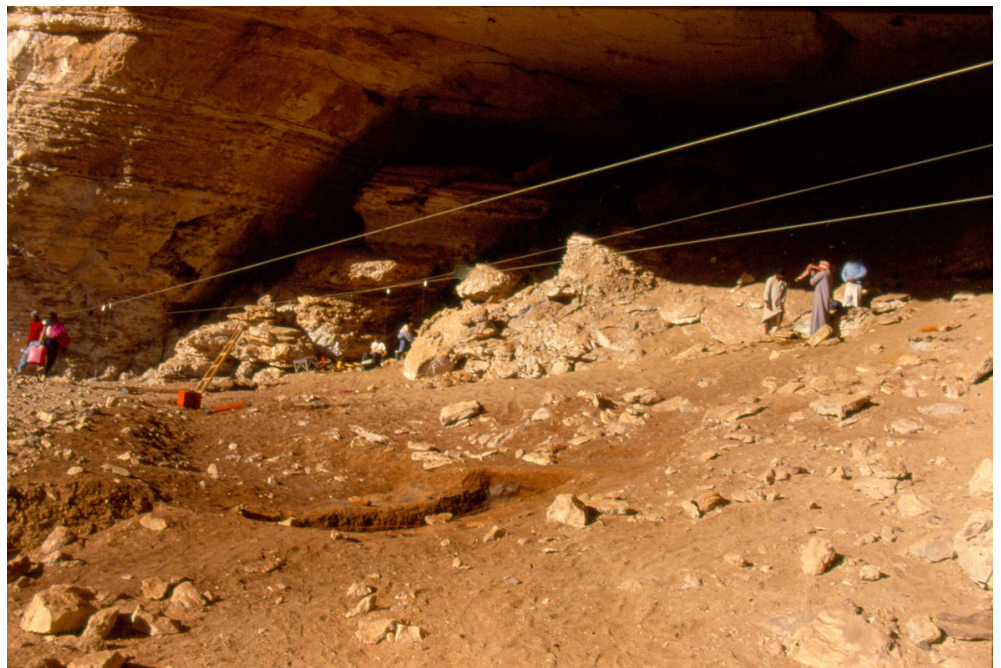

Fig. 5 The surface of the cave interior at the start of the excavations (photo: P. Vermeersch)

sized limestone fragments. The rockfall, at the cave entrance, contains essentially clastsupported angular limestone and relatively abundant chert fragments. The edge of the rockfall wall does not form a single, continuous, steep scree slope. Instead, there is a subhorizontal interfingering of rock debris lobes with the layers coming from inside the cave, thus indicating the episodic nature of the rockfall. In sector A, the backfill is at least $2 \mathrm{~m}$ thick and contains mineralogical as well as organic material. The majority of the organic material consists of intact or mechanically fragmented animal droppings. Successive fills (layers A to D) rest discomformably upon older backfill (Fig. 6) with MSA artefacts and are attributed to the Holocene. The upper layer represents the "gully depression," while the lower one represents the "main archaeological zone." The latter can be easily distinguished on the basis of numerous hearths. The fill of the "gully depression" was rather poor in archaeological material, whereas the "main archaeological zone" was quite rich.

Working from top (most recent) to base, the following stratigraphic sequence has been identified:

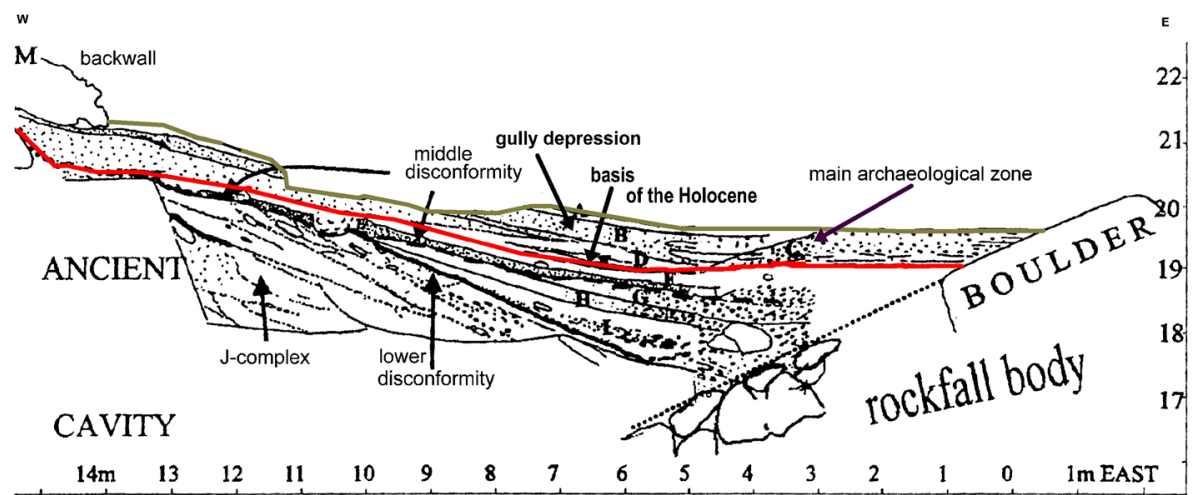

Fig. 6 Stratigraphic section west-east along 23 N (adapted from Moeyersons et al. 2002) 
- Layers A and B: deposits and the archaeological material that fill the gully depression

- Layer C: deposits and the archaeological material of the main archaeological zone, of which the upper part is the C1-horizon (ca. 6.15-6.48 Ka BP) and the lower part is the C2-horizon (ca. 6.68-7.35 Ka BP).

A number of profiles with plotted artefacts and features are presented here in order to illustrate this sequence (Figs. 7 and 8).

\section{Results}

\section{Holocene Features}

In the main archaeological horizon, numerous archaeological features were registered during the excavation. These features are described in chronological order of their excavation (additional data are given in Online Resource 2). Figure 9 gives an overview of the position of each feature within the cave. Table 2 identifies their stratigraphic position. Most features functioned as hearths. They consist very often of white ash covering an accumulation of small or large charcoal fragments, resting upon reddish burnt sediment. They are usually circular or somewhat oval with an average diameter of about $42 \mathrm{~cm}$. The preserved white ash accumulation is often rather restricted in surface and thickness. In some instances, numerous hearthstones are present, such as is the case for features F103, F104, F107 and F203. However, hearths without hearthstone(s) also occur. The principal species represented in the charcoal from the hearths (Table 1) vary. There seems to be no correlation between the feature structure and the main charcoal species present.

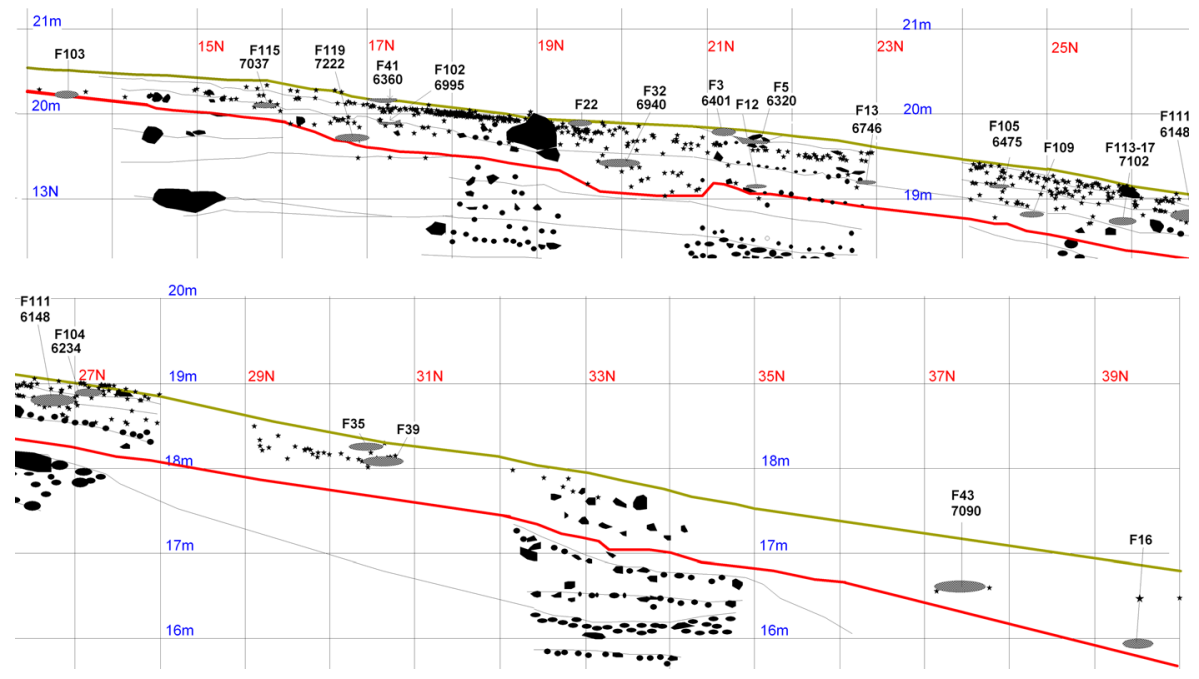

Fig. 7 A composite south-north profile, partially from $4 \mathrm{~W}(14-18 \mathrm{~N}, 24-28 \mathrm{~N})$ with artefacts (stars) plotted from $3.00 \mathrm{~W}$ up to $3.49 \mathrm{~W}$. The hearths from 3 to $4 \mathrm{~W}$ have been positioned on the profile with their feature number and ${ }^{14} \mathrm{C}$ age in BP. The area between the olive and red line corresponds to the Holocene deposits (image in full colour online) 


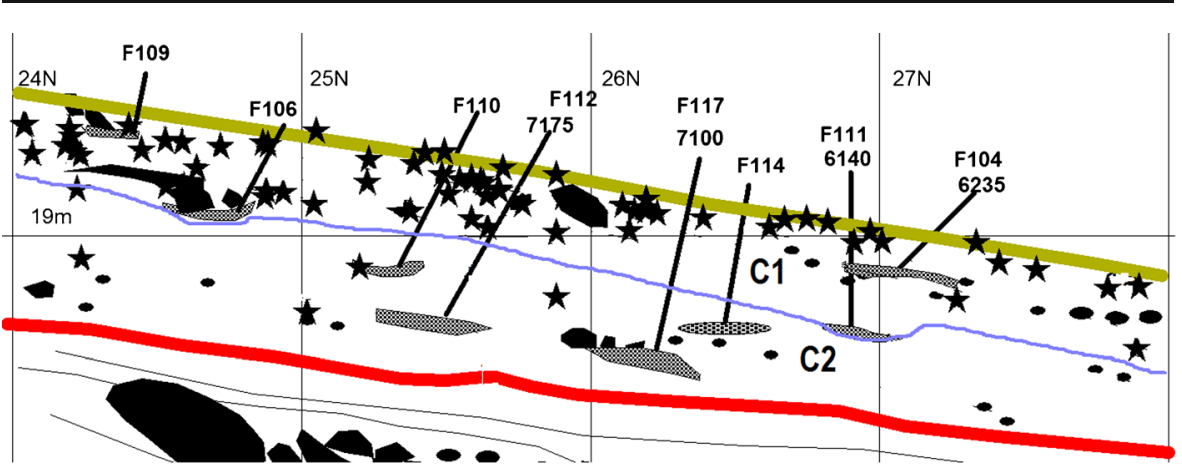

Fig. 8 South-north profile (24-28 N), from $2 \mathrm{~W}$ with artefacts (stars) plotted from $2.00 \mathrm{~W}$ up to $2.49 \mathrm{~W}$

At Tree Shelter, archaeological horizon 3, several hearths (e.g., F12 and F23) were quite similar to some of the hearths (e.g., Feature 104) at Sodmein Cave but in a better state of preservation. Little information is available regarding the activities performed at these hearths.

The artefact distribution (Fig. 10) in the excavated trenches reflects the paucity of artefacts in the gully depression deposits. There seems to be no clear correlation between areas of high artefact density and specific features. The eastern edge of the artefact distribution coincides with the westward expansion of the rockfall wall. The absence of artefacts in 14-17 N $4 \mathrm{~W}$ is not a reality but the result of a registration error in the field.

\section{Radiocarbon Chronology of the Site}

Charcoal was plentiful in all deposits. Several handpicked charcoal samples were submitted for conventional and AMS dating. The resulting dates are listed in uncalibrated form in Table 2. All Sodmein dates were calibrated using the OxCal v4.2.2 software (Bronk Ramsey 2009) (Fig. 11). Feature 311, from near the surface, is clearly of a recent date.

\section{The Holocene Artefacts}

The artefacts collected in the main archaeological horizon are considered to belong to a time period younger than 7.0 Ka cal BC, frequently younger even than $6.25 \mathrm{Ka}$ cal BC and older than $5.15 \mathrm{Ka}$ cal $\mathrm{BC}$. This artefact collection will be treated as a single assemblage, considered the "Holocene" assemblage, even when it is certain that a high number of occupations have been present. It is noteworthy that more than 70 features, most of them hearths or hearth remains, have been registered within the "Holocene" deposits. The few tool types, which are considered as typologically characteristic, will be described individually and related to their stratigraphic and chronological position and, where possible, to a specific feature.

\section{Chert Occurrences}

Good chert is abundantly present in the area of Wadi Sodmein. Most exploited chert nodules have an ovaloid shape and typically consist of a grey-whitish, fine structured chert with good knapping qualities. Sometimes this chert is dotted with white spots, sometimes 


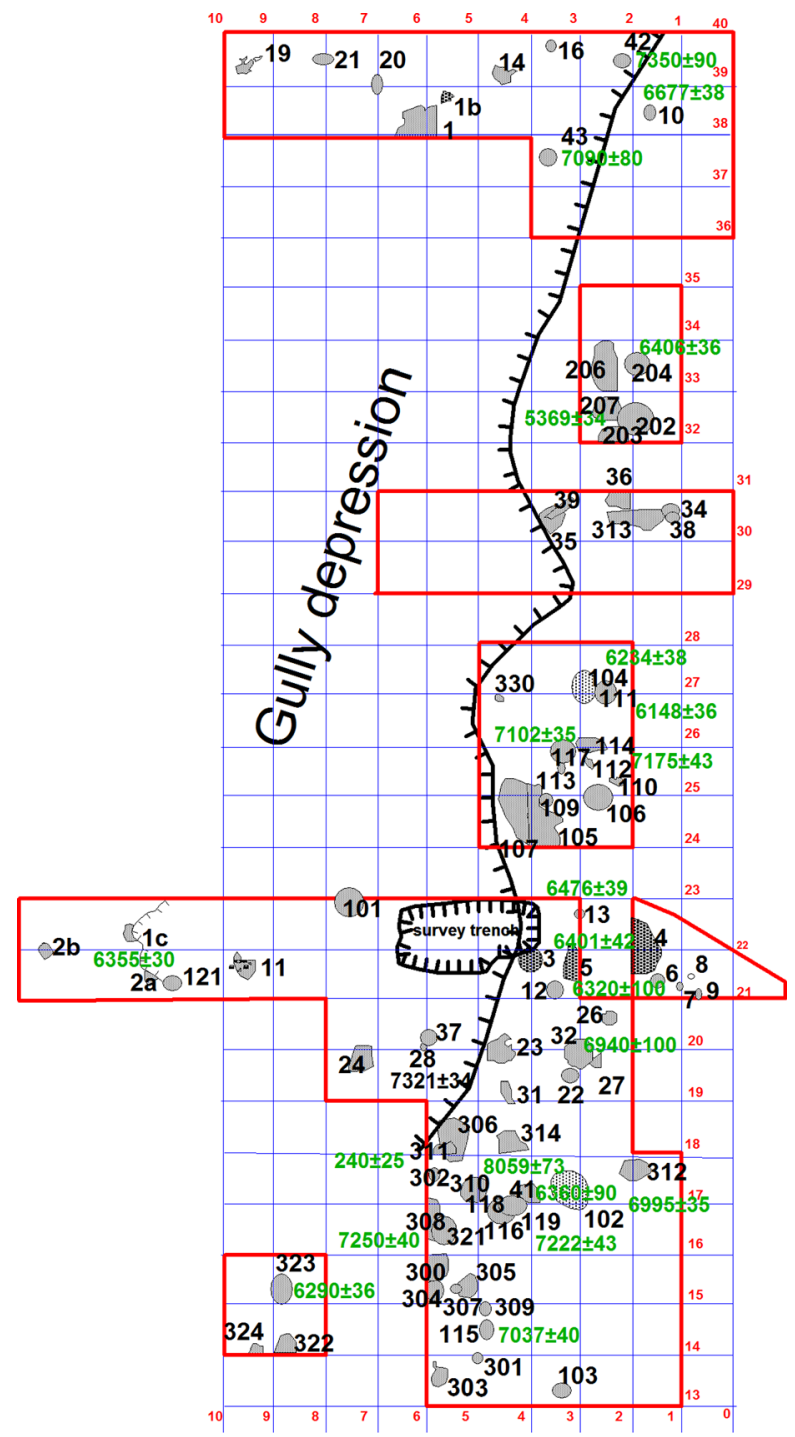

Fig. 9 Plan of features attributed to the Holocene occupation and their ${ }^{14} \mathrm{C}$ age in years BP

it is darker in the centre of the nodule. Less frequently, the chert colour is brownish or even bluish. Flint with a rolled abraded cortex is most common, suggesting they were collected from the wadi deposits. The cortex surface is most often rather smooth with a whitish colour. Artefacts with a fresh cortex are rare. Quartz pebbles were also used for a limited number of products. Such pebbles can also be found in the nearby wadi deposits.

\section{Debitage}

The artefacts are most often preserved in a fresh state with cutting edges intact and are either unpatinated or only slightly patinated. The lithic material (Table 3 ) is composed 
Table 1 Charcoal determinations from some of the features (number of fragments)

\begin{tabular}{|c|c|c|c|c|c|c|c|c|c|c|c|}
\hline \multirow{2}{*}{$\begin{array}{l}\text { Layer } \\
\text { Feature }\end{array}$} & \multirow{2}{*}{$\begin{array}{l}\text { Layer A-B } \\
\text { F1 }\end{array}$} & \multicolumn{5}{|c|}{ Layer C1 } & \multicolumn{5}{|c|}{ Layer C2 } \\
\hline & & F3 & F5 & $\mathrm{F} 31$ & F41 & F10 & F112 & F119 & F6 & F28 & Scattered \\
\hline Acacia $\mathrm{sp}$. & & & 5 & 23 & 97 & & & 25 & 53 & 57 & 35 \\
\hline Salvadora persica & & & 55 & 52 & 48 & & 23 & & 38 & 35 & 5 \\
\hline Tamarix sp. & & & 30 & 11 & 70 & & & & 14 & 86 & 21 \\
\hline Capparis decidua & & & 2 & 4 & 25 & & & & & & \\
\hline Maerua crassifolia & & & & & 17 & & & & & 3 & \\
\hline Fagonia/Zygophyllum & & & & & 1 & & & & 4 & & \\
\hline cf. Suaeda & & & 1 & 2 & & & & & & & \\
\hline Chenopodiaceae & & & & 7 & 13 & & & & 3 & 18 & \\
\hline Fabaceae & & & & & & & & & & 2 & \\
\hline Dicotiledons indet & & & 1 & 4 & 2 & & & & & & \\
\hline Indet. (partly "melted") & 150 & 150 & 4 & 5 & 18 & 150 & & & 8 & 6 & 4 \\
\hline Indet. & & & & 14 & 8 & & & & 9 & & \\
\hline Total studied charcoal fragments & 150 & 150 & 98 & 122 & 299 & 150 & 23 & 25 & 120 & 207 & 65 \\
\hline
\end{tabular}

mainly of flakes, some cortical flakes and smaller debitage remains. Cores for blade production (Fig. 12) are rare. This situation might suggest an import of blades into the cave. Levallois products are present (Fig. 13: 6, 8(?), 11-12), albeit in small quantities. All of them were located in the central or southern parts of the cave and are probably in a secondary position, having being eroded from lower deposits of the Middle Stone Age.

Cores (Table 3) are not well represented, comprising only $1.22 \%$ of the assemblage. Most cores have been formed out of a rolled nodule, although sometimes a large, thick cortical flake was used. Flakes are produced without their removal being preceded by special core preparation. Cortex is often still present. Most cores are exhausted. Direct hard-hammer percussion is the only technique used for the debitage. The cores are mostly of irregular type (Fig. 12: 2). When a more systematic knapping approach was used, a single platform procedure was preferred (Fig. 12: 3-8). In addition to these irregular or single platform cores for flakes, some single platform cores for bladelets (Fig. 12: 6 and 8) are present. Other core types, such as multiplatform cores (Fig. 12: 1) and opposed platform cores (Fig. 12: 9), are rare. One of the multiplatform cores can be interpreted as a thick scraper.

Flakes are numerous, displaying very few traces of preparation. The percussion bulb is usually quite prominent. Flakes have mainly been obtained without preparation of the platform and often have a flat butt (66\%). Sometimes they have a dihedral butt (22\%) (Table 4). Facetted butts are rare. Average flake length was estimated (no measurements were taken) at about $4 \mathrm{~cm}$. Large flakes are rare. In addition to chert, quartz (1\%) was also used, mainly for the production of small flakes (Fig. 17: 11). No quartz cores were found, suggesting that the flakes were introduced into the cave. Cortical flakes and blades are not numerous (about $2 \%$ of the assemblage). Apparently, decortication took place outside the cave and mainly blanks were introduced. Blades (Figs. 13: 1-3, 17: 1) 


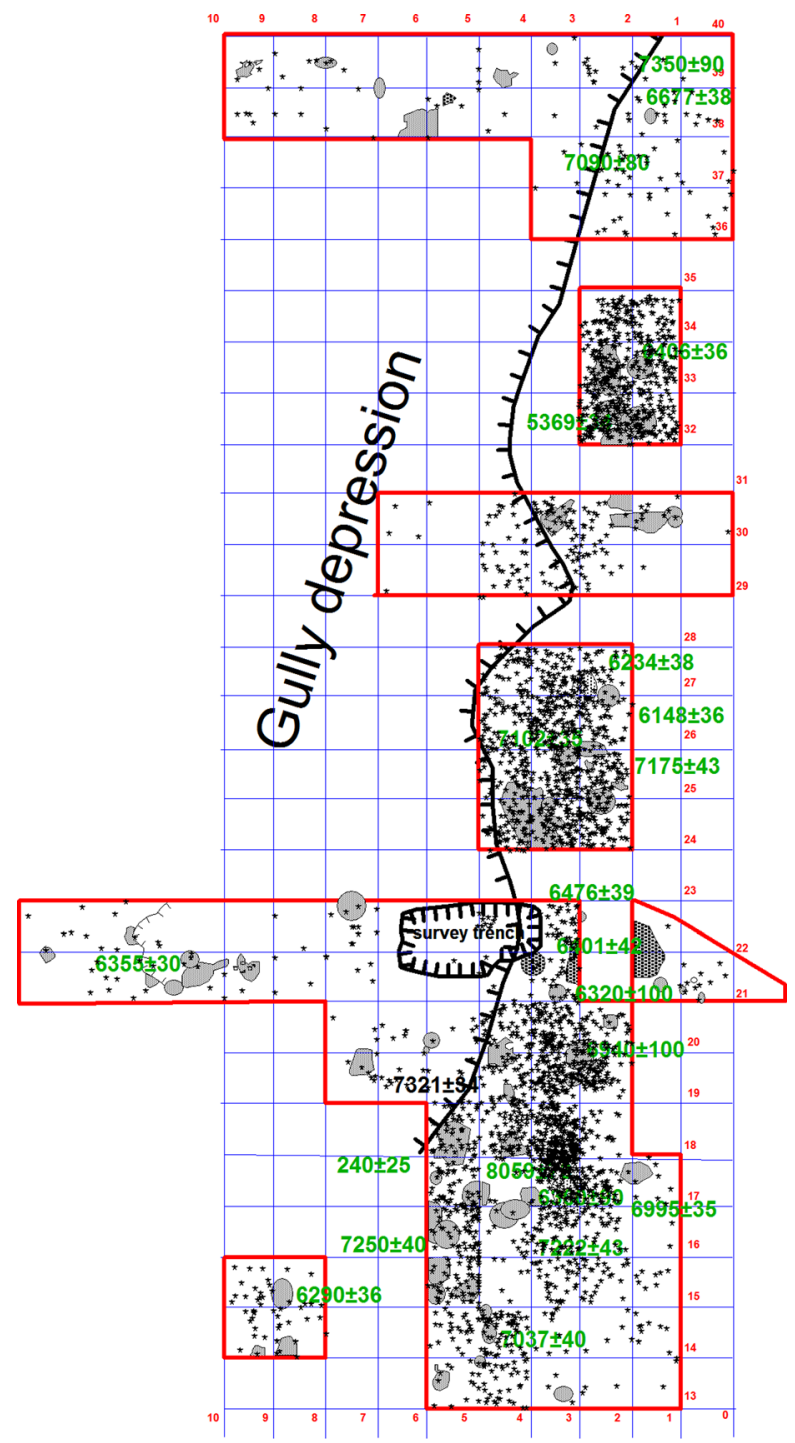

Fig. 10 Plan of artefacts and features

and bladelets are not numerous (about $4.4 \%$ of the assemblage) and certainly not characteristic for the assemblage. They are rather short and thick, not really elongated and probably the incidental result of flake production, and not of a specific blade debitage technique. Thick blades have a triangular section. The butt (Table 4) is mostly flat, usually with a pronounced percussion bulb, suggesting a very restricted core preparation. Blades and bladelets of Epipalaeolithic type (Fig. 15: 3) are rare. Some of the Epipalaeolithic bladelets were used as blanks for microlithic points (Fig. 15: 45). Their presence and the age of Feature 310 (7.1 Ka cal BC) give rise to the hypothesis that groups of Elkabian (Vermeersch 1978, 2012) hunter-gatherers visited the site. However, a specific horizon with Elkabian debitage or tools is not present. It 
Table 2 Features from the Holocene deposits. Sample pre-treatment methods for radiocarbon dating: A - acid extraction, AAA - acid-alkali-acid extraction, $\mathrm{H}_{2} \mathrm{~S}_{4}$ - acid treatment of carbonate samples (see Supporting Information 2) for detailed method descriptions

\begin{tabular}{|c|c|c|c|c|c|c|c|c|}
\hline FeatureF & Position & Elevation & Layer & $\begin{array}{l}\text { Dated } \\
\text { material }\end{array}$ & Sample ID & Age BP & Method & Pretreat. \\
\hline 311 & $18.2 \mathrm{~N} 5.5 \mathrm{~W}$ & 20.27 & Surf. & $\mathrm{Ch}$ & ME99/45/96 & $240 \pm 25$ (KIA-28777) & AMS & AAA \\
\hline 303 & $13.6 \mathrm{~N} 5.7 \mathrm{~W}$ & 20.77 & A-B & & & & & \\
\hline 301 & $13.9 \mathrm{~N} 4.9 \mathrm{~W}$ & 20.65 & A-B & & & & & \\
\hline 302 & $17.6 \mathrm{~N} 5.9 \mathrm{~W}$ & 20.67 & A-B & & & & & \\
\hline 312 & $17.6 \mathrm{~N} 1.9 \mathrm{~W}$ & 19.97 & A-B & & & & & \\
\hline 24 & $19.3 \mathrm{~N} 7.3 \mathrm{~W}$ & 19.90 & A-B & & & & & \\
\hline 26 & $20.6 \mathrm{~N} 2.4 \mathrm{~W}$ & 20.10 & A-B & & & & & \\
\hline 121 & $21.4 \mathrm{~N} 11.0 \mathrm{~W}$ & 21.70 & A-B & & & & & \\
\hline $2 \mathrm{~b}$ & $22.0 \mathrm{~N} 13.5 \mathrm{~W}$ & 21.10 & & & & & & \\
\hline 11 & 2136 N $9.5 \mathrm{~W}$ & 20.10 & A-B & & & & & \\
\hline 101 & $22.9 \mathrm{~N} 7.5 \mathrm{~W}$ & 19.29 & A-B & & & & & \\
\hline 35 & $30.5 \mathrm{~N} 3.6 \mathrm{~W}$ & 18.20 & A-B & & & & & \\
\hline 202 & $32.4 \mathrm{~N} 1.9 \mathrm{~W}$ & 17.99 & A-B & & & & & \\
\hline 207 & $32.6 \mathrm{~N} 2.6 \mathrm{~W}$ & 17.64 & A-B & $\mathrm{Ch}$ & ME95/123 & $5369 \pm 34(\mathrm{COL} 1337.1 .2)$ & AMS & AAA \\
\hline $1 b$ & $38.6 \mathrm{~N} 5.4 \mathrm{~W}$ & 17.30 & A-B & & & & & \\
\hline 1 & 38.7 N $5.9 \mathrm{~W}$ & 17.44 & A-B & & & & & \\
\hline 20 & $38.9 \mathrm{~N} 7.0 \mathrm{~W}$ & 18.00 & A-B & & & & & \\
\hline 19 & $39.3 \mathrm{~N} 9.4 \mathrm{~W}$ & 18.57 & A-B & & & & & \\
\hline 21 & $39.5 \mathrm{~N} 8.0 \mathrm{~W}$ & 18.00 & A-B & & & & & \\
\hline 322 & $14.2 \mathrm{~N} 8.6 \mathrm{~W}$ & 21.10 & $\mathrm{C} 1$ & & & & & \\
\hline 324 & $14.0 \mathrm{~N} 9.4 \mathrm{~W}$ & 21.34 & $\mathrm{C} 1$ & & & & & \\
\hline 323 & $15.3 \mathrm{~N} 8.8 \mathrm{~W}$ & 21.09 & $\mathrm{C} 1$ & $\mathrm{Ch}$ & ME99/49/44 & $6290 \pm 36($ COL1339.1.2) & AMS & AAA \\
\hline 116 & $16.0 \mathrm{~N} 4.7 \mathrm{~W}$ & 20.06 & $\mathrm{C} 1$ & & & & & \\
\hline 41 & $17.2 \mathrm{~N} 4.0 \mathrm{~W}$ & 20.18 & $\mathrm{C} 1$ & $\mathrm{Ch}$ & ME93/508 & $6360 \pm 90(\mathrm{Lv}-2085)$ & Conv. & A \\
\hline 22 & $19.5 \mathrm{~N} 3.2 \mathrm{~W}$ & 19.94 & $\mathrm{C} 1$ & & & & & \\
\hline 27 & $19.8 \mathrm{~N} 2.7 \mathrm{~W}$ & 19.84 & $\mathrm{C} 1$ & & & & & \\
\hline 23 & $20.0 \mathrm{~N} 4.6 \mathrm{~W}$ & 19.83 & $\mathrm{C} 1$ & & & & & \\
\hline 31 & $19.2 \mathrm{~N} 4.4 \mathrm{~W}$ & 19.45 & $\mathrm{C} 1$ & & & & & \\
\hline 3 & $21.8 \mathrm{~N} 4.0 \mathrm{~W}$ & 19.62 & $\mathrm{C} 1$ & $\mathrm{Ch}$ & ME93/05 & $6401 \pm 40(\mathrm{COL} 1325.1 .1)$ & AMS & AAA \\
\hline $2 \mathrm{a}$ & $21.4 \mathrm{~N} 11.4 \mathrm{~W}$ & 20.70 & $\mathrm{C} 1$ & OES & ME93/69 & $6354 \pm 31(\mathrm{COL} 1326.1 .2)$ & AMS & $\mathrm{H}_{2} \mathrm{SO}_{4}$ \\
\hline $1 \mathrm{c}$ & $22.4 \mathrm{~N} 11.9 \mathrm{~W}$ & 20.65 & $\mathrm{C} 1$ & & & & & \\
\hline 5 & $21.7 \mathrm{~N} 3.0 \mathrm{~W}$ & 19.60 & $\mathrm{C} 1$ & $\mathrm{Ch}$ & ME93/31 & $6320 \pm 100(\mathrm{Lv}-2082)$ & Conv. & A \\
\hline 105 & $24.5 \mathrm{~N} 3.7 \mathrm{~W}$ & 19.15 & $\mathrm{C} 1$ & $\mathrm{Ch}$ & ME95/15/406 & $6475 \pm 36(\mathrm{COL} 1330.1 .2)$ & AMS & AAA \\
\hline 111 & $27.1 \mathrm{~N} 2.5 \mathrm{~W}$ & 18.63 & $\mathrm{C} 1$ & $\mathrm{Ch}$ & ME95/17/598 & $6148 \pm 36(\mathrm{COL} 1331.1 .1)$ & AMS & AAA \\
\hline 330 & $26.9 \mathrm{~N} 4.6 \mathrm{~W}$ & 18.98 & $\mathrm{C} 1$ & & & & & \\
\hline 104 & $27.3 \mathrm{~N} 2.9 \mathrm{~W}$ & 18.90 & $\mathrm{C} 1$ & $\mathrm{Ch}$ & ME95/15/299 & $6234 \pm 38($ COL1329.1.2) & AMS & AAA \\
\hline 36 & $30.9 \mathrm{~N} 2.2 \mathrm{~W}$ & 18.10 & $\mathrm{C} 1$ & & & & & \\
\hline 39 & $30.4 \mathrm{~N} 3.5 \mathrm{~W}$ & 18.10 & $\mathrm{C} 1$ & & & & & \\
\hline 203 & $32.4 \mathrm{~N} 2.2 \mathrm{~W}$ & 17.94 & $\mathrm{C} 1$ & & & & & \\
\hline 204 & $33.5 \mathrm{~N} 1.9 \mathrm{~W}$ & 17.70 & $\mathrm{C} 1$ & $\mathrm{Ch}$ & ME95/97 & $6406 \pm 36(\mathrm{COL} 1336.1 .2)$ & AMS & AAA \\
\hline 103 & $13.1 \mathrm{~N} 3.2 \mathrm{~W}$ & 20.36 & $\mathrm{C} 2$ & & & & & \\
\hline 115 & $14.4 \mathrm{~N} 4.8 \mathrm{~W}$, & 20.19 & $\mathrm{C} 2$ & $\mathrm{Ch}$ & ME95/11/84 & $7037 \pm 40($ COL1333.1.1) & AMS & AAA \\
\hline 305 & $15.4 \mathrm{~N} 5.1 \mathrm{~W}$ & 20.40 & $\mathrm{C} 2$ & & & & & \\
\hline
\end{tabular}


Table 2 (continued)

\begin{tabular}{|c|c|c|c|c|c|c|c|c|}
\hline FeatureF & Position & Elevation & Layer & $\begin{array}{l}\text { Dated } \\
\text { material }\end{array}$ & Sample ID & Age BP & Method & Pretreat. \\
\hline 300 & $15.0 \mathrm{~N} 5.5 \mathrm{~W}$ & 20.56 & $\mathrm{C} 2$ & & & & & \\
\hline 304 & $15.4 \mathrm{~N} 5.8 \mathrm{~W}$ & 20.44 & $\mathrm{C} 2$ & & & & & \\
\hline 307 & $15.4 \mathrm{~N} 5.5 \mathrm{~W}$ & 20.35 & $\mathrm{C} 2$ & & & & & \\
\hline 119 & $16.9 \mathrm{~N} 4.3 \mathrm{~W}$ & 19.66 & $\mathrm{C} 2$ & $\mathrm{Ch}$ & ME95/18/1 & $7222 \pm 43(\operatorname{COL} 1335.1 .1)$ & AMS & AAA \\
\hline 308 & 16.9 N $5.9 \mathrm{~W}$ & 20.10 & $\mathrm{C} 2$ & $\mathrm{Ch}$ & ME99/46/15 & $7250 \pm 40$ (IRPA-1372) & AMS & AAA \\
\hline 118 & $17.1 \mathrm{~N} 4.8 \mathrm{~W}$ & 19.78 & $\mathrm{C} 2$ & & & & & \\
\hline 102 & $17.3 \mathrm{~N} 3.2 \mathrm{~W}$ & 20.00 & $\mathrm{C} 2$ & $\mathrm{Ch}$ & ME95/10/149 & $6995 \pm 35$ (KIA-28778) & AMS & AAA \\
\hline 306 & $18.3 \mathrm{~N} 5.5 \mathrm{~W}$ & 20.05 & $\mathrm{C} 2$ & & & & & \\
\hline 314 & $18.2 \mathrm{~N} 4.3 \mathrm{~W}$ & 19.81 & $\mathrm{C} 2$ & & & & & \\
\hline 32 & $19.9 \mathrm{~N} 3.1 \mathrm{~W}$ & 19.43 & $\mathrm{C} 2$ & $\mathrm{Ch}$ & ME93/366 & $6940 \pm 100(\mathrm{Lv}-2083)$ & Conv. & $\mathrm{A}$ \\
\hline 28 & $20.0 \mathrm{~N} 6.1 \mathrm{~W}$ & 19.70 & $\mathrm{C} 2$ & $\mathrm{Ch}$ & ME93/315 & $7321 \pm 36($ COL1328.1.2) & AMS & AAA \\
\hline 12 & $21.0 \mathrm{~N} 3.8 \mathrm{~W}$ & 19.15 & $\mathrm{C} 2$ & & & & & \\
\hline 9 & $21.1 \mathrm{~N} 0.7 \mathrm{~W}$ & 19.40 & $\mathrm{C} 2$ & & & & & \\
\hline 6 & $21.2 \mathrm{~N} 1.0 \mathrm{~W}$ & 19.58 & $\mathrm{C} 2$ & & & & & \\
\hline 7 & $21.4 \mathrm{~N} 1.1 \mathrm{~W}$ & 19.41 & $\mathrm{C} 2$ & & & & & \\
\hline 8 & $21.4 \mathrm{~N} 0.7 \mathrm{~W}$ & 19.39 & $\mathrm{C} 2$ & & & & & \\
\hline 4 & $21.9 \mathrm{~N} 2.0 \mathrm{~W}$ & 19.65 & $\mathrm{C} 2$ & & & & & \\
\hline 13 & $22.7 \mathrm{~N} 3.0 \mathrm{~W}$ & 19.14 & $\mathrm{C} 2$ & $\mathrm{Ch}$ & ME93/545 & $6746 \pm 39(\mathrm{COL} 1341.1 .2)$ & AMS & AAA \\
\hline 109 & $24.7 \mathrm{~N} 3.7 \mathrm{~W}$ & 18.82 & $\mathrm{C} 2$ & & & & & \\
\hline 106 & $24.9 \mathrm{~N} 2.6 \mathrm{~W}$ & 19.16 & $\mathrm{C} 2$ & & & & & \\
\hline 107 & $24.9 \mathrm{~N} 2.6 \mathrm{~W}$ & 19.11 & $\mathrm{C} 2$ & & & & & \\
\hline 110 & $25.2 \mathrm{~N} 2.4 \mathrm{~W}$ & 18.91 & $\mathrm{C} 2$ & & & & & \\
\hline 114 & $25.5 \mathrm{~N} 3.0 \mathrm{~W}$ & 18.72 & $\mathrm{C} 2$ & & & & & \\
\hline 113 & $25.5 \mathrm{~N} 3.5 \mathrm{~W}$ & 18.71 & $\mathrm{C} 2$ & & & & & \\
\hline 112 & $25.7 \mathrm{~N} 2.8 \mathrm{~W}$ & 18.73 & $\mathrm{C} 2$ & $\mathrm{Ch}$ & ME95/17/646 & $7175 \pm 42(\mathrm{COL} 1332.1 .1)$ & AMS & AAA \\
\hline 117 & $25.7 \mathrm{~N} 3.4 \mathrm{~W}$ & 18.61 & $\mathrm{C} 2$ & $\mathrm{Ch}$ & ME95/17/655 & $7102 \pm 35(\mathrm{COL} 1334.1 .2)$ & AMS & AAA \\
\hline 34 & $30.7 \mathrm{~N} 1.3 \mathrm{~W}$ & 18.17 & $\mathrm{C} 2$ & & & & & \\
\hline 38 & $30.4 \mathrm{~N} 1.1 \mathrm{~W}$ & 18.05 & $\mathrm{C} 2$ & & & & & \\
\hline 206 & $32.5 \mathrm{~N} 2.5 \mathrm{~W}$ & 17.52 & $\mathrm{C} 2$ & & & & & \\
\hline 43 & $37.5 \mathrm{~N} 3.5 \mathrm{~W}$ & 16.42 & $\mathrm{C} 2$ & $\mathrm{Ch}$ & ME93/428 & $7090 \pm 80(\mathrm{Lv}-2086)$ & Conv. & $\mathrm{A}$ \\
\hline 10 & $38.4 \mathrm{~N} 1.6 \mathrm{~W}$ & 16.83 & $\mathrm{C} 2$ & $\mathrm{Ch}$ & ME93/61 & $6677 \pm 38(\mathrm{COL} 1327.1 .2)$ & AMS & $\mathrm{AAA}$ \\
\hline 42 & $39.5 \mathrm{~N} 2.1 \mathrm{~W}$ & 16.10 & $\mathrm{C} 2$ & $\mathrm{Ch}$ & ME93/333 & $7350 \pm 80(\mathrm{UtC}-3312)$ & AMS & $\mathrm{AAA}$ \\
\hline 310 & $17.3 \mathrm{~N} 5.2 \mathrm{~W}$ & 19.63 & $\mathrm{C} 2$ base & $\mathrm{Ch}$ & ME99/46/15 & $8059 \pm 73($ COL1338.1.2) & AMS & AAA \\
\hline
\end{tabular}

Ch charcoal, OES ostrich egg shell

seems that remains of the Epipalaeolithic Elkabian were eroded before the arrival of later groups.

\section{Retouched Blanks}

Tools (Table 5), defined as retouched blanks, are well represented (6\% of the artefacts). Simple tools, such as retouched flakes (34\%), denticulates (18\%) and notched flakes (6\%), are by far the most dominant categories. However, typologically more specified 


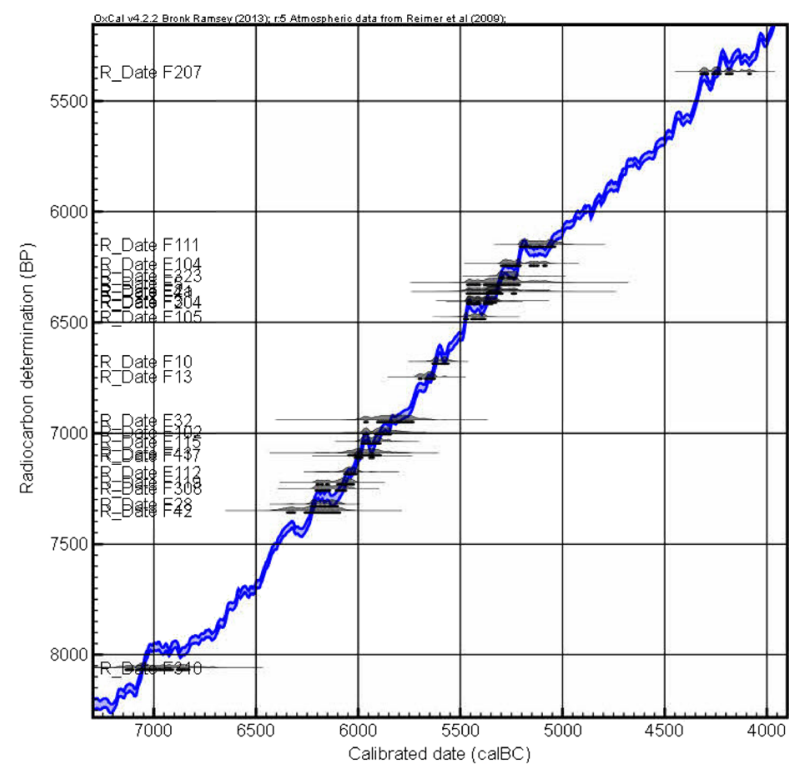

Fig. 11 Calibration of the radiocarbon dates of the Sodmein Cave using OxCal v4.2.2

tools such as different types of arrowheads (together about $11 \%$ ) are also well represented.

End-scrapers are present but they do not represent particularly specific types. Some are thumbnail-scrapers (Fig. 15: 26-27); others are end-scrapers on an elongated flake (Fig. 15: 25, 28) or on a thick flake. Some of the end-scrapers have a denticulated scraper front (Fig. 17: 8), display a bifacial retouch (Fig. 15: 24) or are only end-scraper fragments (Fig. 14: 16). There is a large cortical scraper (Fig. 14: 5) which resembles scrapers that occur often in predynastic and Neolithic contexts, such as Djara A (Gehlen et al. 2002). Some side-scrapers (Fig. 13: 4) display an MSA morphology and could be intrusive from the lower levels. Borers are present but not numerous. Some are obtained by two distal notches (Fig. 13: 7) or by a more significant distal retouch (Fig. 16: 1-3). One of them (Fig. 16: 1), with an intensively used tip, is produced on a flake by semiabrupt to abrupt retouch. A large borer (Fig. 16: 5) resembles those found in the Badarian (Hendrickx et al. 2001). Only two burins (Fig. 14: 1, 6), originating from the northern sector $\mathrm{C} 1$-deposits, have been discovered. Bifacial flaking is clearly present, but most bifacial pieces are fragments (Fig. 16: 6) or seem to be preforms (Fig. 16: 9, 10). They can be interpreted as fragments of bifacial knives. Their form is not the result of careful specialised production. A small, more carefully prepared, bifacial foliate (Fig. 15: 29) was found in the A-deposits. Most bifacial artefacts are less characteristic and often display a restricted bifacial retouch.

Several arrowhead types are present. Some of them are similar to Epipalaeolithic or early Neolithic types such as Ounan points. Two Ounan points (Fig. 15: 4, 5) are similar to those recovered at Abu Tartur 1072 (Riemer et al. 2004). They have been manufactured on pointed (Epipalaeolithic) bladelets which have obtained a central stem by a steep retouch. A pointed backed bladelet (Fig. 15: 6) can also be attributed to Epipalaeolithic activity. Most arrowheads are characterised by a rather flat, invasive 
Table 3 Debitage products from the Holocene assemblages

\begin{tabular}{|c|c|c|c|c|c|c|c|}
\hline \multirow[t]{2}{*}{ ID } & \multirow{2}{*}{$\begin{array}{l}\text { A-B } \\
N\end{array}$} & \multicolumn{2}{|l|}{ C 1} & \multicolumn{2}{|l|}{$\mathrm{C} 2$} & \multicolumn{2}{|l|}{ Total } \\
\hline & & $N$ & $\%$ & $N$ & $\%$ & $N$ & $\%$ \\
\hline Single platform core for flakes & 1 & 8 & 0.23 & 6 & 0.27 & 15 & 0.26 \\
\hline Single platform core for blade(let)s & 0 & 3 & 0.09 & 0 & 0 & 3 & 0.05 \\
\hline Opposed platform core & 1 & 1 & 0.03 & 4 & 0.18 & 6 & 0.11 \\
\hline Multiplatform core & 1 & 0 & 0 & 3 & 0.14 & 4 & 0.07 \\
\hline Discoid core & 0 & 1 & 0.03 & 1 & 0.05 & 2 & 0.04 \\
\hline Irregular core & 0 & 18 & 0.52 & 10 & 0.46 & 28 & 0.49 \\
\hline Core preform & 0 & 3 & 0.09 & 6 & 0.27 & 9 & 0.16 \\
\hline Core fragment & 0 & 1 & 0.03 & 0 & 0 & 1 & 0.02 \\
\hline Core rejuvenation & 0 & 0 & 0 & 1 & 0.05 & 1 & 0.02 \\
\hline Core tablet & 0 & 0 & 0 & 2 & 0.09 & 2 & 0.04 \\
\hline Crested flake & 0 & 0 & 0 & 1 & 0.05 & 1 & 0.02 \\
\hline Cortical flake & 0 & 81 & 2.33 & 25 & 1.14 & 106 & 1.86 \\
\hline Cortical flake fragment & 0 & 3 & 0.09 & 15 & 0.69 & 18 & 0.32 \\
\hline Cortical blade & 0 & 2 & 0.06 & 0 & 0 & 2 & 0.04 \\
\hline Cortical blade fragment & 0 & 0 & 0 & 2 & 0.09 & 2 & 0.04 \\
\hline Flake & 12 & 1343 & 38.58 & 1200 & 54.87 & 2555 & 44.89 \\
\hline Flake fragment & 4 & 684 & 19.65 & 209 & 9.56 & 897 & 15.76 \\
\hline Chip & 2 & 1015 & 29.16 & 317 & 14.49 & 1334 & 23.44 \\
\hline Quartz flake & 0 & 57 & 1.64 & 17 & 0.78 & 74 & 1.30 \\
\hline Quartz chip & 0 & 5 & 0.14 & 1 & 0.05 & 6 & 0.11 \\
\hline Chunk & 1 & 20 & 0.57 & 9 & 0.41 & 30 & 0.53 \\
\hline Levallois preparation flake & 0 & 1 & 0.03 & 0 & 0 & 1 & 0.02 \\
\hline Levallois flake & 0 & 0 & 0 & 2 & 0.09 & 2 & 0.04 \\
\hline (Levallois) retouched flake & 0 & 2 & 0.06 & 8 & 0.37 & 10 & 0.18 \\
\hline (Levallois) point fragment & 0 & 1 & 0.03 & 1 & 0.05 & 2 & 0.04 \\
\hline Blade & 0 & 31 & 0.89 & 72 & 3.29 & 103 & 1.81 \\
\hline Blade irregular & 0 & 4 & 0.11 & 0 & 0 & 4 & 0.07 \\
\hline Blade fragment & 0 & 3 & 0.09 & 2 & 0.09 & 5 & 0.09 \\
\hline Blade fragment distal & 0 & 6 & 0.17 & 0 & 0 & 6 & 0.11 \\
\hline Blade fragment proximal & 0 & 11 & 0.32 & 28 & 1.28 & 39 & 0.69 \\
\hline Blade fragment medial & 0 & 9 & 0.26 & 44 & 2.01 & 53 & 0.93 \\
\hline Blade fragment distal & 0 & 5 & 0.14 & 3 & 0.14 & 8 & 0.14 \\
\hline Bladelet & 0 & 2 & 0.06 & 21 & 0.96 & 23 & 0.40 \\
\hline Bladelet fragment proximal & 0 & 0 & 0 & 1 & 0.05 & 1 & 0.02 \\
\hline Bladelet fragment medial & 0 & 0 & 0 & 1 & 0.05 & 1 & 0.02 \\
\hline Bladelet fragment distal & 0 & 0 & 0 & 1 & 0.05 & 1 & 0.02 \\
\hline Epipalaeo bladelet & 0 & 1 & 0.03 & 0 & 0 & 1 & 0.02 \\
\hline Tool & 2 & 160 & 4.6 & 174 & 7.96 & 336 & 5.90 \\
\hline Total & 24 & 3481 & 100 & 2187 & 100 & 5692 & 100.00 \\
\hline
\end{tabular}



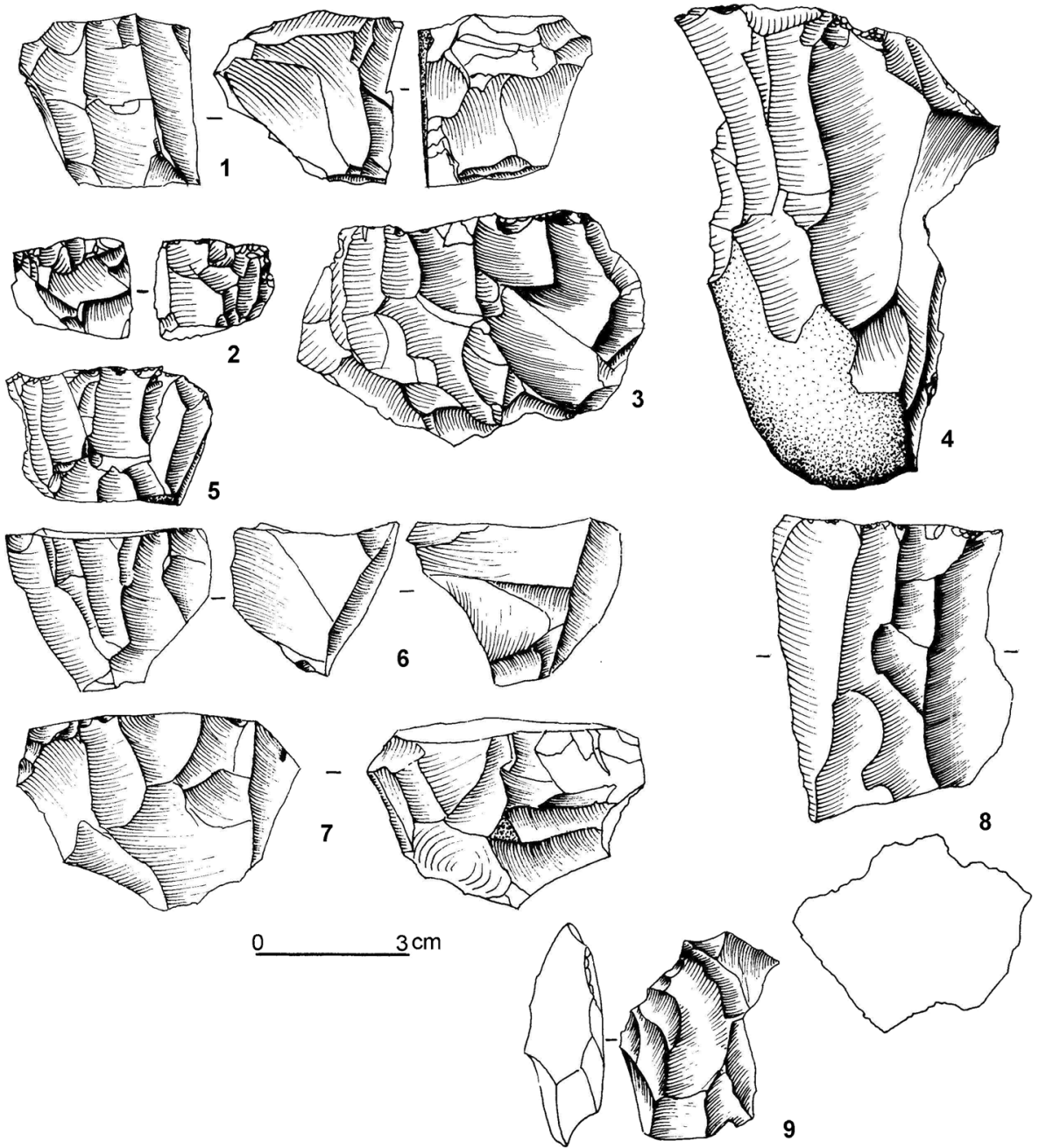

Fig. 12 Cores. 1: multiplatform core; 2: irregular core; 3-8: single platform core; 9: opposed platform core

retouch. They often display a lenticular form, generally with a bifacial retouch (Fig. 15: $11,13-15,18)$ on a flake. Occasionally. the surfaces display a covering retouch (Fig. 15: 18). An irregular trapeze (Fig. 15: 23), which might have been a transverse arrowhead, was present.

Other tools or tool fragments are rare. An axe preparation flake (Fig. 14: 8) was found in the upper part of the Holocene levels. A cortical flake fragment with very flat retouch (Fig. 14: 13) is similar to some of the predynastic retouched flakes. Most other tools are uncharacteristic and comprise a number of diverse retouched blades and blade fragments (Fig. 13: 1, 2-3, 5), some of which attest a fine retouch (Fig. 13: 3). Retouched flakes and their fragments are among the most frequent tool types. They are diversified in their appearance (Figs. 13: 2, 10; 14: 7, 11, 15; 15: 30; 16: 4, 11, 16; 17: 9, 13; 18: 4). Some of the retouched flakes (Fig. 14: 11, 13 and 15) have flat retouches, which often occur on predynastic tools. Notched flakes (Figs. 13: 9; 17: 5; 

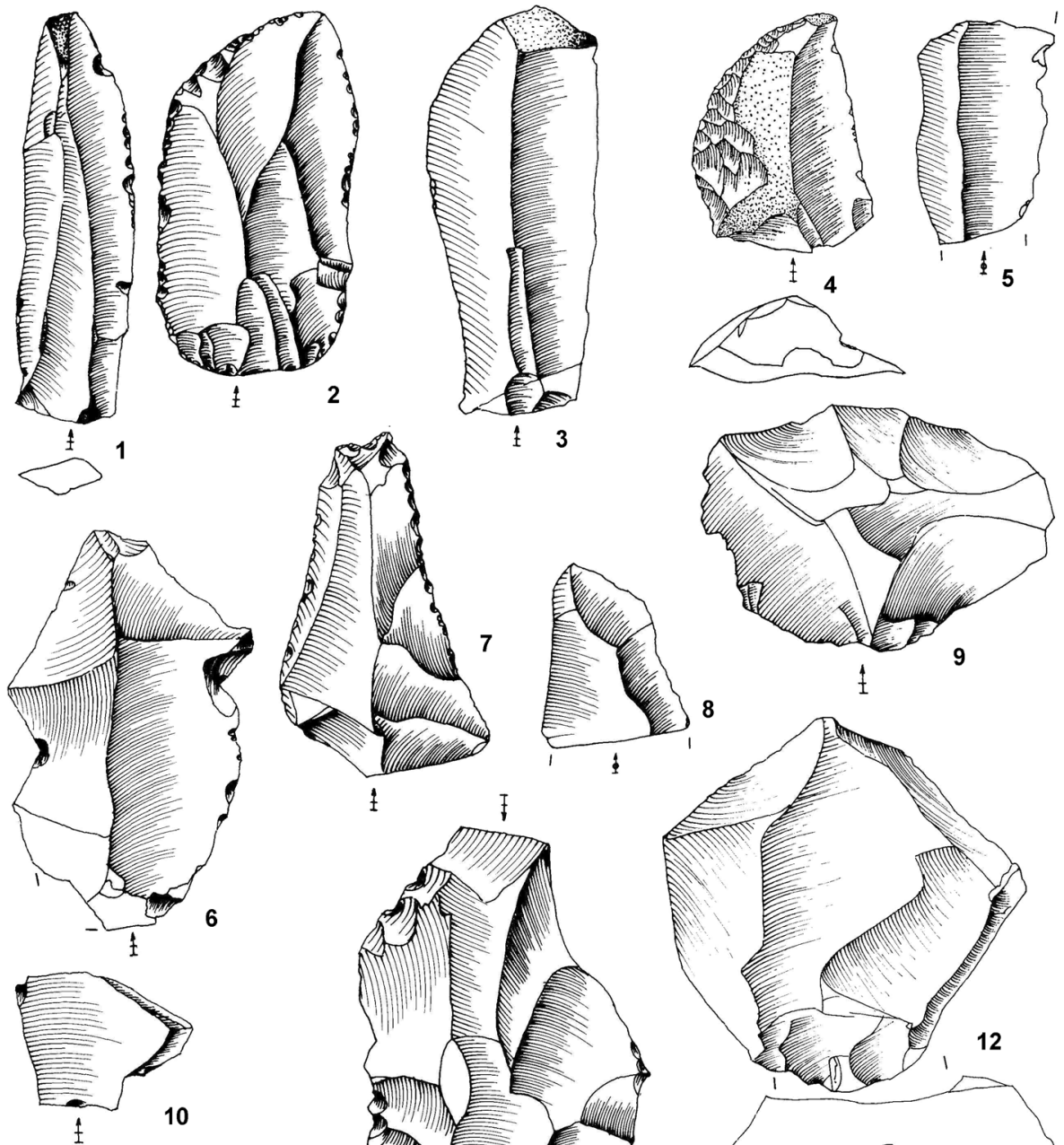

$\underline{0}$ $3 \mathrm{~cm}$
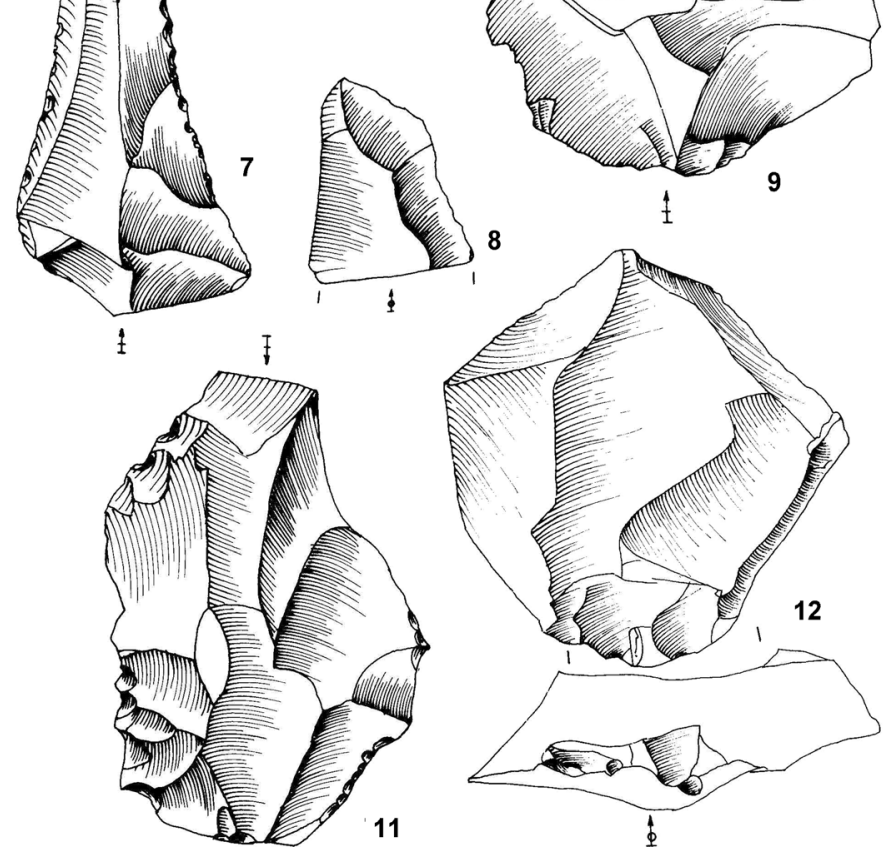

Fig. 13 Debitage products. 1-2: retouched blade; 3: blade with fine retouch; 4: side-scraper; 5: retouched blade fragment; 6: retouched (Levallois) flake; 7: retouched flake or borer; 8: (Levallois) point fragment; 9: notched flake; 10: retouched flake; 11: retouched (Levallois?) flake; 12: Levallois flake

Table 4 Butt-type distribution of blades/bladelets

\begin{tabular}{llllll}
\hline & Flat & Dihedral & Pointed & Facetted & Diverse \\
\hline Blades/bladelets & 16 & 2 & 1 & 1 & 1 \\
Flakes & 644 & 214 & 22 & 0 & 89 \\
$\%$ flakes & 66.46 & 22.08 & 2.27 & 0 & 9.18 \\
\hline
\end{tabular}


Table 5 Tools from the Holocene assemblages

\begin{tabular}{|c|c|c|c|c|c|c|c|}
\hline \multirow[t]{2}{*}{ ID } & \multirow{2}{*}{$\begin{array}{l}\text { A-B } \\
N\end{array}$} & \multicolumn{2}{|c|}{ C 1} & \multicolumn{2}{|l|}{$\mathrm{C} 2$} & \multicolumn{2}{|l|}{ Total } \\
\hline & & $N$ & $\%$ & $N$ & $\%$ & $N$ & $\%$ \\
\hline End-scraper & 0 & 3 & 1.9 & 7 & 3.9 & 10 & 2.9 \\
\hline End-scraper fragment & 0 & 1 & 0.6 & 1 & 0.6 & 2 & 0.6 \\
\hline Denticulated end-scraper & 0 & 1 & 0.6 & 5 & 2.8 & 6 & 1.8 \\
\hline Thumbnail-scraper & 1 & 1 & 0.6 & 2 & 1.1 & 4 & 1.2 \\
\hline Bifacial end-scraper & 0 & 0 & 0.0 & 1 & 0.6 & 1 & 0.3 \\
\hline Side-scraper & 0 & 2 & 1.3 & 2 & 1.1 & 4 & 1.2 \\
\hline Predynastic cortical scraper & 0 & 1 & 0.6 & 1 & 0.6 & 2 & 0.6 \\
\hline Borer & 0 & 3 & 1.9 & 2 & 1.1 & 5 & 1.5 \\
\hline Badarian large borer & 0 & 1 & 0.6 & 1 & 0.6 & 2 & 0.6 \\
\hline Burin on break & 0 & 1 & 0.6 & 0 & 0.0 & 1 & 0.3 \\
\hline Burin on natural surface & 0 & 1 & 0.6 & 0 & 0.0 & 1 & 0.3 \\
\hline Backed blade & 0 & 1 & 0.6 & 1 & 0.6 & 2 & 0.6 \\
\hline Backed bladelet & 0 & 1 & 0.6 & 0 & 0.0 & 1 & 0.3 \\
\hline Denticulate & 0 & 23 & 14.6 & 32 & 17.7 & 55 & 16.1 \\
\hline Denticulate fragment & 0 & 5 & 3.2 & 0 & 0.0 & 5 & 1.5 \\
\hline Denticulated blade & 0 & 2 & 1.3 & 4 & 2.2 & 6 & 1.8 \\
\hline Denticulated blade frag. ventral retouch & 0 & 1 & 0.6 & 1 & 0.6 & 2 & 0.6 \\
\hline Denticulated chip flake & 0 & 1 & 0.6 & 0 & 0.0 & 1 & 0.3 \\
\hline Fine denticulate & 0 & 0 & 0.0 & 3 & 1.7 & 3 & 0.9 \\
\hline Arrowhead & 0 & 0 & 0.0 & 6 & 3.3 & 6 & 1.8 \\
\hline Arrowhead fragment & 0 & 0 & 0.0 & 1 & 0.6 & 1 & 0.3 \\
\hline Arrowhead preform & 0 & 2 & 1.3 & 7 & 3.9 & 9 & 2.6 \\
\hline Point tang fragment & 0 & 1 & 0.6 & 0 & 0.0 & 1 & 0.3 \\
\hline Pointed flake & 0 & 1 & 0.6 & 0 & 0.0 & 1 & 0.3 \\
\hline Irregular trapeze & 0 & 1 & 0.6 & 0 & 0.0 & 1 & 0.3 \\
\hline Ounan point & 0 & 1 & 0.6 & 2 & 1.1 & 3 & 0.9 \\
\hline Stemmed arrowhead or borer & 0 & 1 & 0.6 & 0 & 0.0 & 1 & 0.3 \\
\hline Tanged point & 0 & 2 & 1.3 & 0 & 0.0 & 2 & 0.6 \\
\hline Tayac point & 0 & 0 & 0.0 & 3 & 1.7 & 3 & 0.9 \\
\hline Bifacial arrowhead & 0 & 1 & 0.6 & 8 & 4.4 & 9 & 2.6 \\
\hline Bifacial preform & 0 & 0 & 0.0 & 4 & 2.2 & 4 & 1.2 \\
\hline Bifacial fragment & 0 & 1 & 0.6 & 1 & 0.6 & 2 & 0.6 \\
\hline Axe (tranchet) preparation flake & 0 & 0 & 0.0 & 1 & 0.6 & 1 & 0.3 \\
\hline Small flake axe & 0 & 0 & 0.0 & 2 & 1.1 & 2 & 0.6 \\
\hline Retouched blade & 0 & 2 & 1.3 & 2 & 1.1 & 4 & 1.2 \\
\hline Blade with fine retouch & 0 & 0 & 0.0 & 1 & 0.6 & 1 & 0.3 \\
\hline Inverse denticulated blade & 0 & 1 & 0.6 & 1 & 0.6 & 2 & 0.6 \\
\hline Notched bladelet & 0 & 0 & 0.0 & 1 & 0.6 & 1 & 0.3 \\
\hline Predynastic retouched flake & 0 & 2 & 1.3 & 1 & 0.6 & 3 & 0.9 \\
\hline Retouched flake & 0 & 63 & 39.9 & 64 & 35.4 & 127 & 37.2 \\
\hline
\end{tabular}


Table 5 (continued)

\begin{tabular}{|c|c|c|c|c|c|c|c|}
\hline \multirow[t]{2}{*}{ ID } & \multirow{2}{*}{$\begin{array}{l}\text { A-B } \\
N\end{array}$} & \multicolumn{2}{|l|}{ C 1} & \multicolumn{2}{|l|}{ C 2} & \multicolumn{2}{|c|}{ Total } \\
\hline & & $N$ & $\%$ & $N$ & $\%$ & $N$ & $\%$ \\
\hline Retouched flake overpassed & 0 & 1 & 0.6 & 0 & 0.0 & 1 & 0.3 \\
\hline Retouched flake fragment & 1 & 13 & 8.2 & 1 & 0.6 & 15 & 4.4 \\
\hline Flake with inverse retouch & 0 & 1 & 0.6 & 1 & 0.6 & 2 & 0.6 \\
\hline Notched flake & 0 & 12 & 7.6 & 6 & 3.3 & 18 & 5.3 \\
\hline Notched quartz flake & 0 & 1 & 0.6 & 0 & 0.0 & 1 & 0.3 \\
\hline Inversely notched flake & 0 & 0 & 0.0 & 1 & 0.6 & 1 & 0.3 \\
\hline Inversely retouched flake fragment & 0 & 0 & 0.0 & 1 & 0.6 & 1 & 0.3 \\
\hline Flake with blunted edge & 0 & 2 & 1.3 & 2 & 1.1 & 4 & 1.2 \\
\hline Pièce esquillée & 0 & 0 & 0.0 & 1 & 0.6 & 1 & 0.3 \\
\hline Sum tools & 2 & 158 & 100.0 & 181 & 100.0 & 341 & 100.0 \\
\hline
\end{tabular}

18: 3) and denticulated flakes (Figs. 14: 3-4, 9, 12; 16: 8, 12-14; 17: 3, 6, 12, 15) are numerous. Sometimes, retouch is inversely applied (Fig. 16: 7, 17). Some rare denticulated blade and blade fragments (Fig. 17: 2, 4, 10) were found.

The numerous ${ }^{14} \mathrm{C}$ dates suggest that artefacts from the $\mathrm{C} 2$ horizon can be dated to a period from about 6.2 to $5.6 \mathrm{Ka}$ cal $\mathrm{BC}$, whereas those from the $\mathrm{C} 1$ horizon may be assigned to the period from 5.4 to $5.0 \mathrm{Ka}$ cal BC. The age of the artefacts from the youngest deposits is rather uncertain but a general terminus to the occupation may be placed around 4.3 $\mathrm{Ka}$ cal $\mathrm{BC}$.

All the arrowheads were collected in the upper part of the Holocene levels with the exception of four items (Fig. 15: 1, 15, 19-20) that appear to be rather short triangular rough-outs. They are leaf-shaped and only partially bifacial. As they occur from archaeological horizon $\mathrm{C} 2$ onwards, it may be concluded that they were in use from at least $6.2 \mathrm{Ka}$ cal BC.

\section{Ceramic Fabrics (Additional Data are Provided in Online Resource 3)}

In the material collected from within the cave, different types of ceramic fabric can be distinguished. Nearly all of this material is in the form of very small sherds. Few rims and no base fragments were recorded.

Most sherds were collected in the northern part of the cave and mostly in the A and the C1-deposits, where they can be associated with feature 111, which has an age of $6148 \pm 38 \mathrm{BP}$. The concentration of ceramic sherds in squares $25-27 \mathrm{~N} 2-4 \mathrm{~W}$ is mainly composed of a grey and a light red fabric (Fig. 19). It is almost impossible, because of the significant vertical scattering of the artefacts, to associate other sherds with specific features or tools. Many ceramic fragments are associated with the A-deposits, suggesting that they belong to a period posterior to $4.2 \mathrm{Ka}$ cal $\mathrm{BC}$ date obtained from feature 207 situated at the basis of the A-deposits. As there are no absolute age indications for the end of the deposition of the A-deposits; a terminus ante quem is not available. 


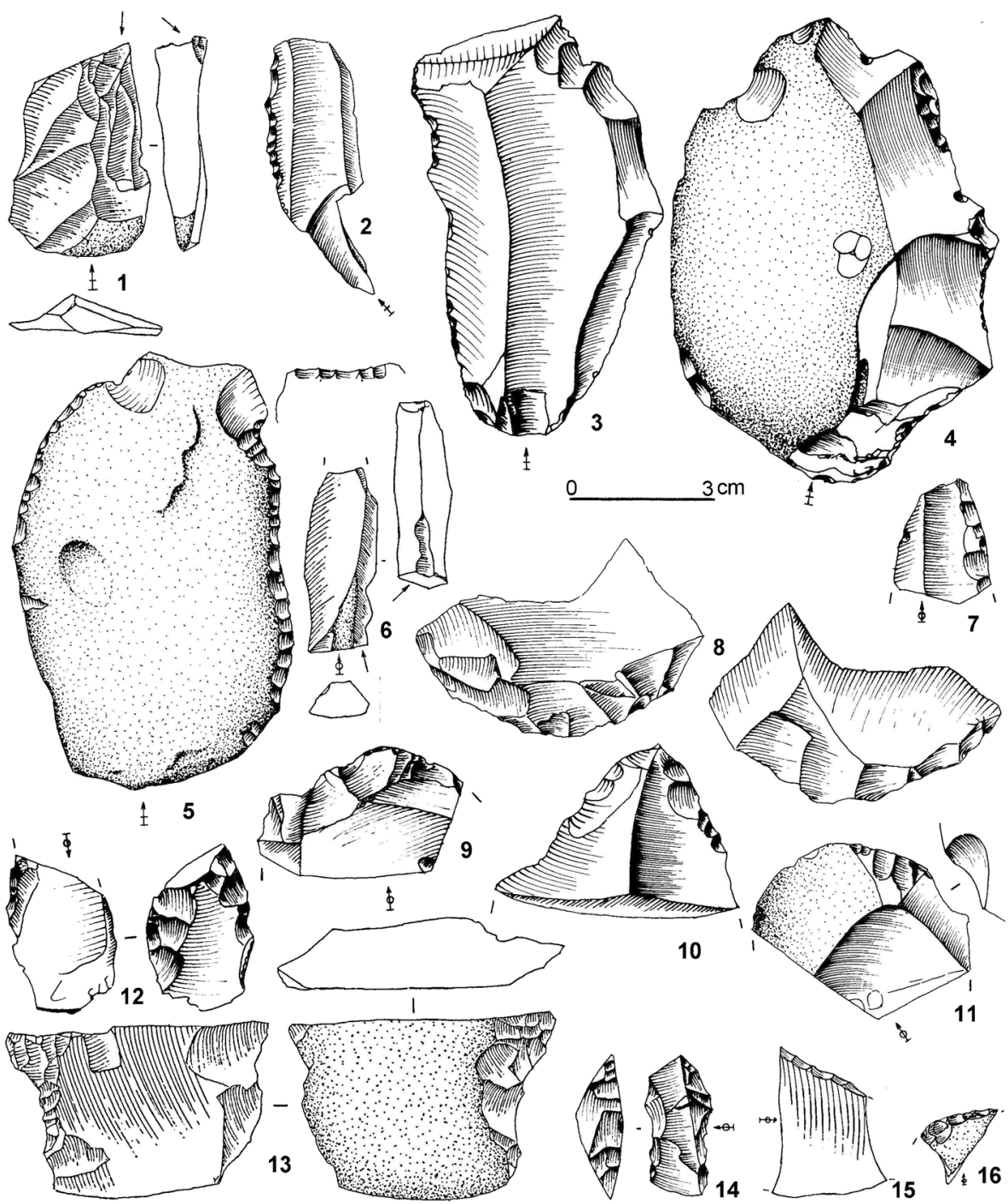

Fig. 14 Retouched blanks. 1: burin on natural surface; 2: retouched blade; 3, 4, 9, 12: denticulated flake; 5: predynastic (?) cortical scraper; 6: burin on a break; 7, 11, 15: retouched flake fragment; 8: axe (tranchet) preparation flake; 10: pointed flake; 13: predynastic (?) retouched flake; 14: small flake axe; 16: end-scraper fragment

\section{Faunal Remains}

Since the publication of the initial results from the Holocene deposits at Sodmein, which mention the presence of caprine remains (Vermeersch et al. 1994), the site has often been cited in the context of the early spread of domesticated caprines in northeastern Africa and the African continent in general. While a report analysing the large amounts of animal dung found in the Sodmein Cave has in the meantime also become available (Linseele et al. 2010), a full description of the bone remains has never been published (Table 6). In view of their importance, the present report on Holocene 

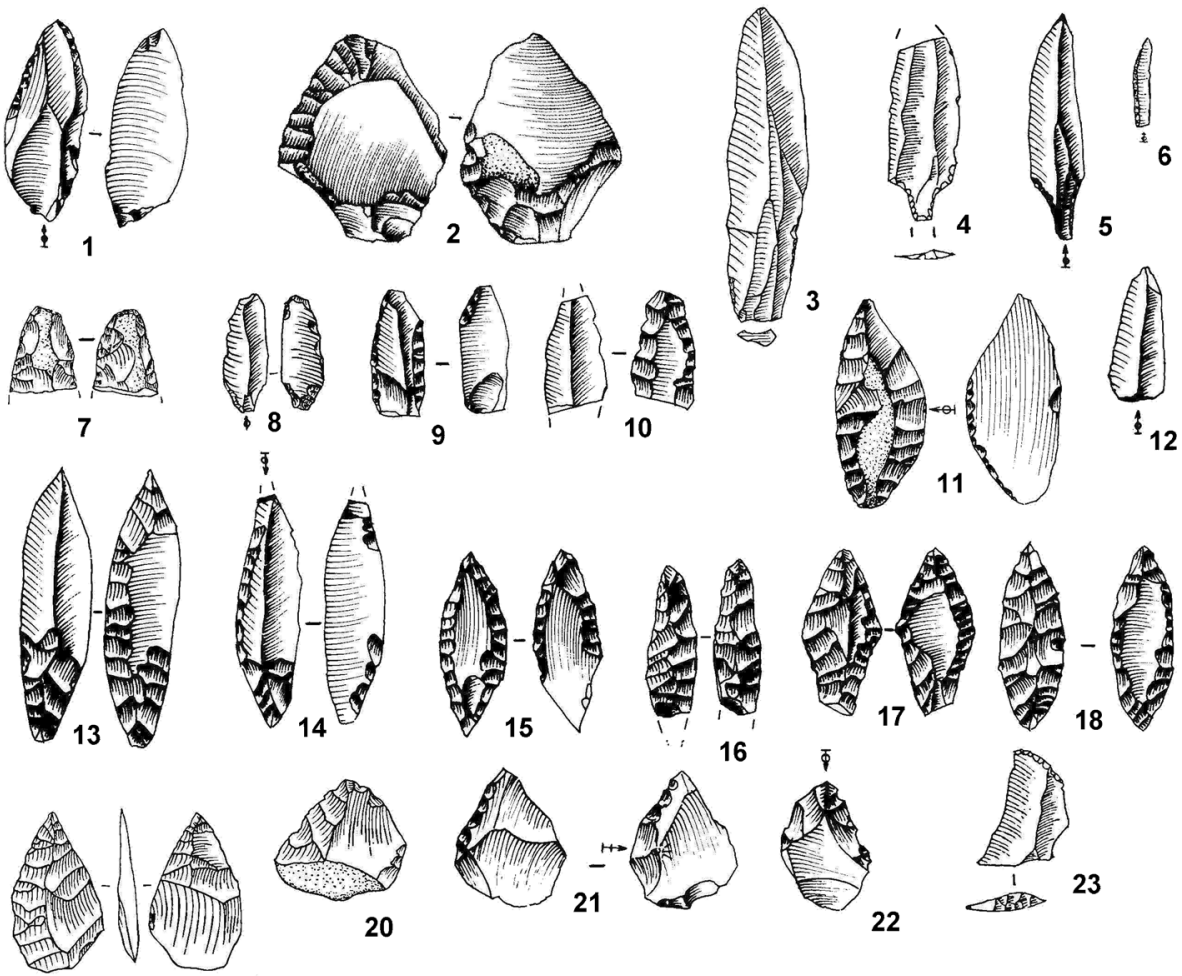

20
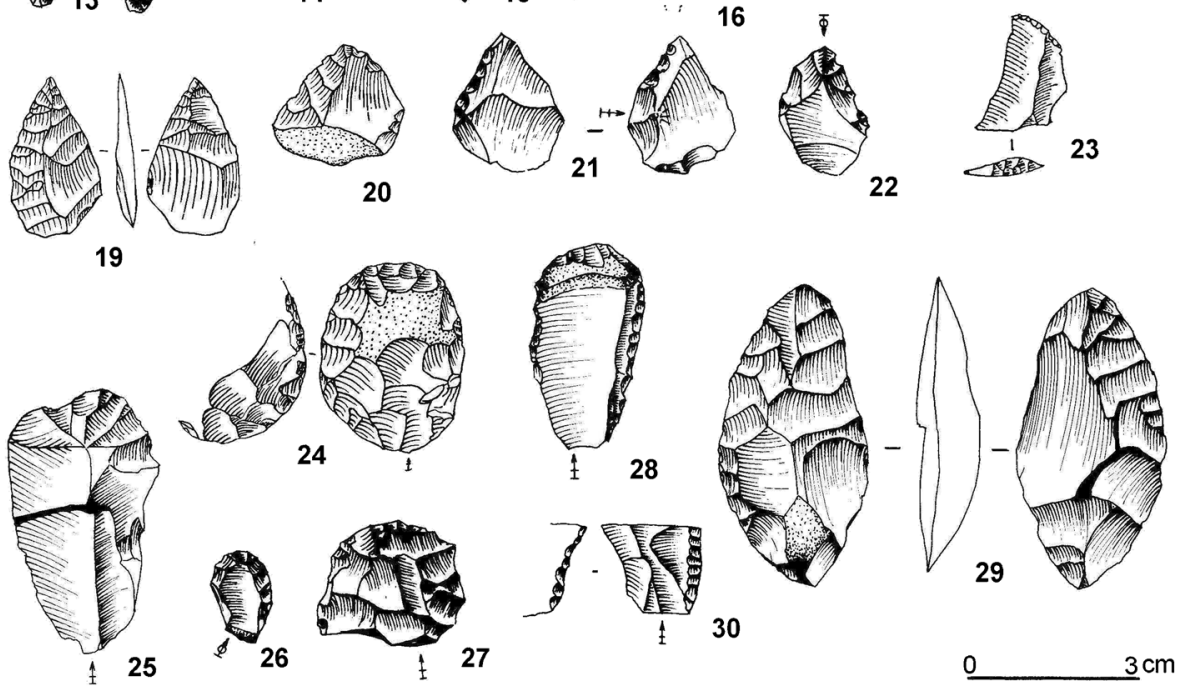

Fig. 15 Retouched blanks. 1: arrowhead; 2, 20-22: arrowhead preform; 3: epipalaeolithic bladelet; 4-5: Ounan point; 6: backed bladelet; 7: bifacial arrowhead fragment; 8: stemmed arrowhead; 9-10: arrowhead preform; 11-12: arrowhead; 13-16, 18-19: bifacial arrowhead; 17: arrowhead (stemmed); 23: irregular trapeze; 24: bifacial end-scraper; 25, 28: end-scraper on a flake; 26-27: thumbnail-scraper; 29: bifacial foliate; 30: retouched flake

Sodmein will therefore provide the necessary details on the actual osteological evidence for domesticates (additional data are given in Online Resource 4).

Most of the faunal remains are presumably non-anthropogenic introductions to the cave, representing animals that may have lived and died there independently of its human occupation. These include various small taxa, the landsnails, amphibians, small lizards and snakes, birds, microbats and rodents. As shown by the find of a pellet with the remains of a minimum of three rodent individuals, small animals may also have 

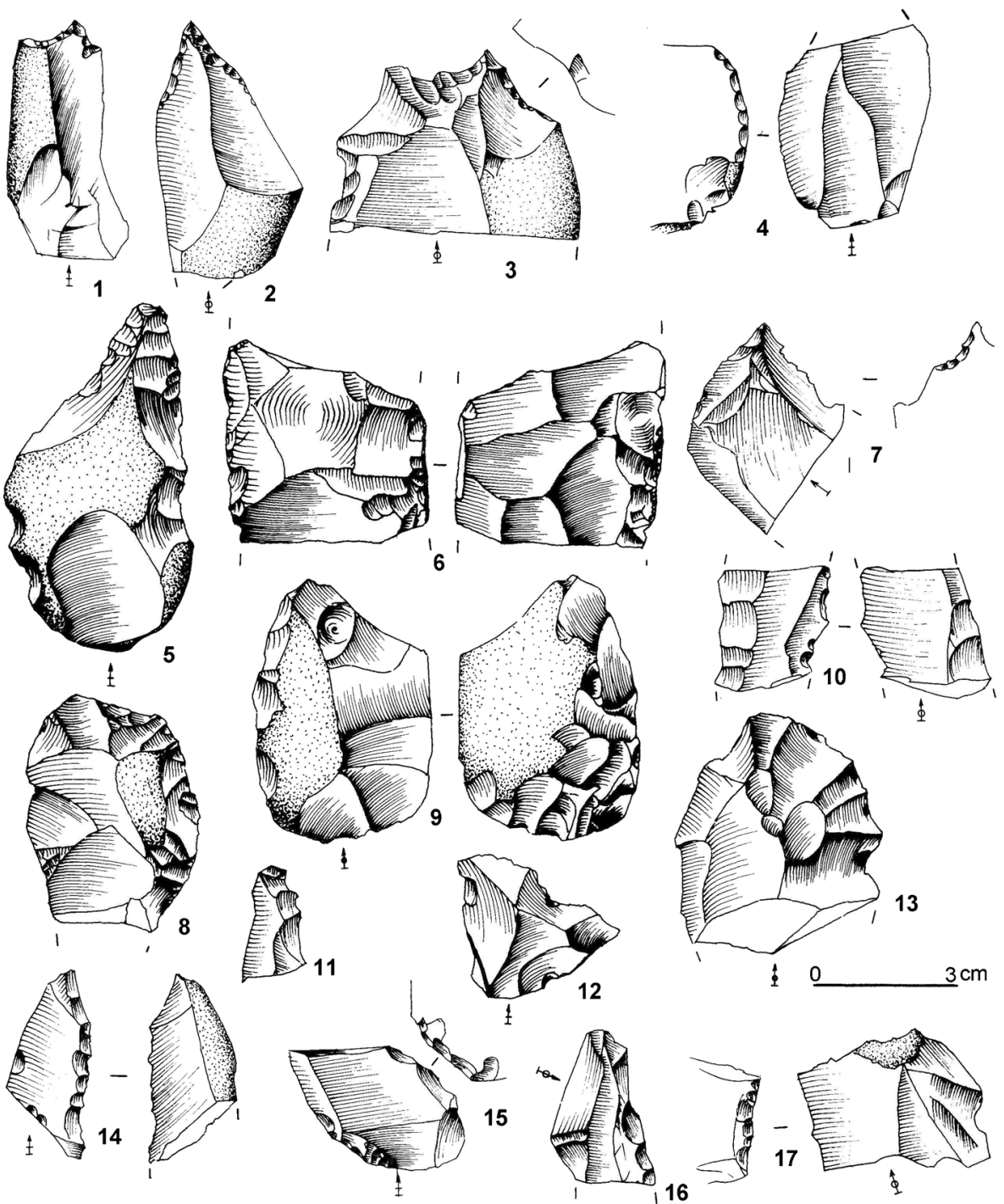

Fig. 16 Retouched blanks. 1-2: borer; 3: notched flake or borer; 4, 11, 16: retouched flake; 5: large borer (Badarian?); 6: bifacial fragment; 7: inversely notched flake; 8, 12-14: denticulated flake; 9-10: bifacial preform; 15: retouched flake fragment or arrowhead preform; 17: inversely retouched flake fragment

ended up in the cave through the activities of birds of prey. Animals that may have been partly hunted by humans include hare, rock dassie, cat, Rüppell's fox, dorcas gazelle and possibly other small wild bovids, for example ibex (Capra ibex) or Barbary sheep (Ammotragus lervia). The find of 30 bones of presumably a single fox, suggests we are dealing with an individual that used the cave and died there without human interference, rather than with a hunted animal. The only fauna with a more probable anthropic origin are the Red Sea shells (Dentalium and cowry), the ostrich eggshell fragments and the domesticated caprines. 

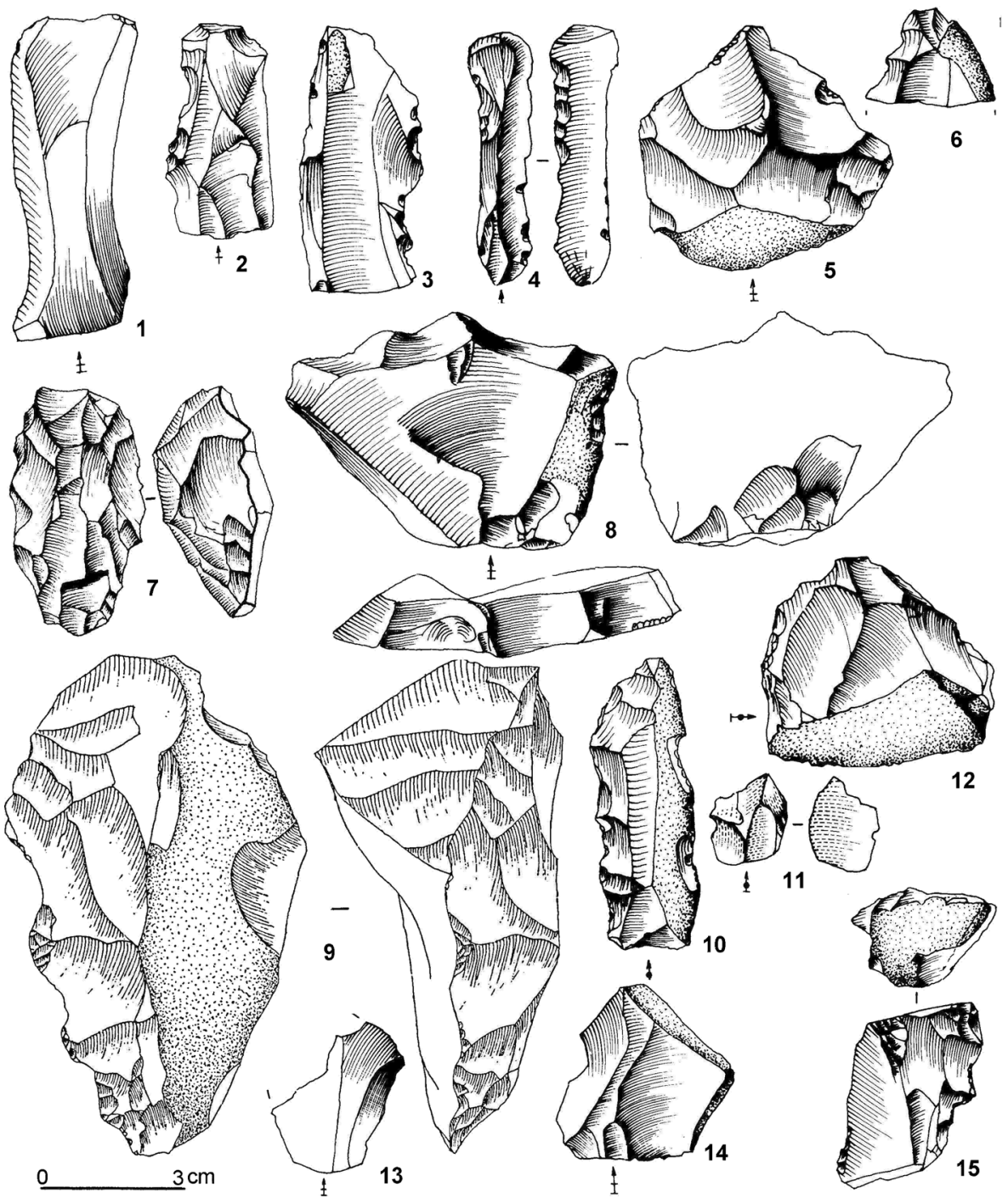

Fig. 17 Retouched blanks. 1: blade; 2: denticulated blade; 3: denticulated flake; 4, 10: denticulated blade; 5: notched flake; 6 : denticulated flake fragment; 7 : multiplatform core or thick scraper; 8 : denticulated endscraper; 9: retouched plunging flake; 11: quartz flake; 12, 15: denticulated flake; 13: retouched flake; 14: notched flake

Osteomorphological and osteometric criteria for the differentiation of sheep, goat, ibex and Barbary sheep, described in Gabler (1985), were used as much as possible but frequently could not be applied on the more small and fragmentary remains. Attributions were generally done on the basis of morphology corresponding to sheep, goat, Barbary sheep or ibex, combined with small size, which allowed a wild species to be excluded. The domesticated caprines total only 10 bones for the entire Holocene sequence.

It is important to observe that the sheep/goat phalanx 1 (Fig. 20, $\mathrm{k}$ and 1, ME99/01/ 252 and ME99/47/15; see Online Resource 4) is associated (same position) with feature 


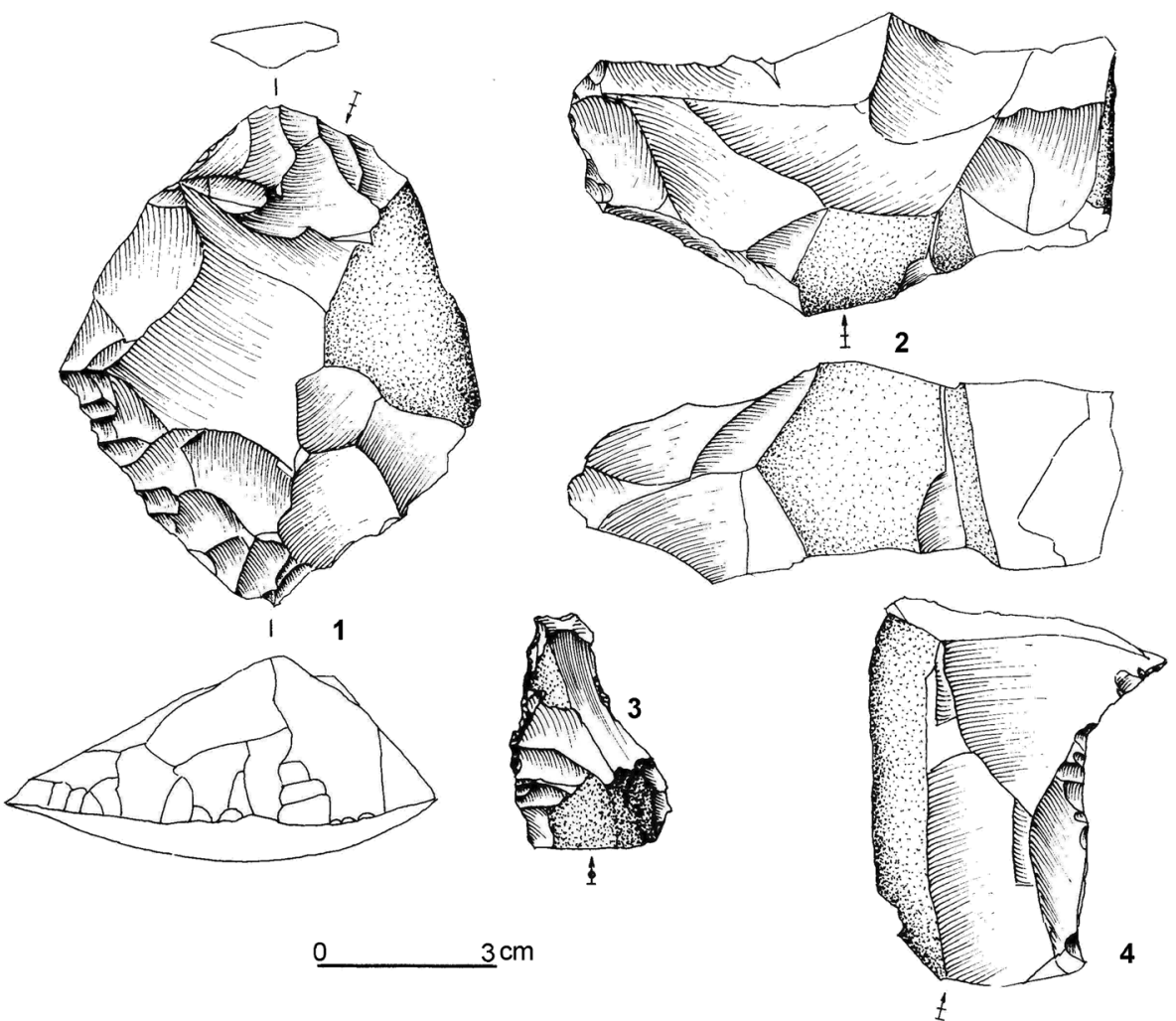

Fig. 18 Retouched blanks. 1: thick scraper; 2: irregular core; 3: notched flake; 4: retouched flake

306 (Online Resource 2), which is clearly below feature 308 stratigraphically (Fig. OR2/33 and Fig. OR4/1) with a date of $7250 \pm 40 \mathrm{BP}(6.2-6.0 \mathrm{Ka}$ Cal BC). The sheep/ goat humerus (ME99/12/36) and os femoris (ME/12/54) (Fig. 20e and f) are situated on a higher level (C1) and associated with feature 105 (Fig. 7 and fig. OR4/1), which has a ${ }^{14} \mathrm{C}$ date of $6475 \pm 35 \mathrm{BP}$. From the stratigraphic position, it is clear that caprines are present from before $7250 \mathrm{BP}$.

The earliest evidence for caprines in the Western Desert was found at Hidden Valley site in Farafra Oasis, in contexts dated to $7251 \pm 67$ BP (Gautier 2014). This means that Sodmein yielded the oldest caprines so far known from the African continent, introduced from at least $6.2 \mathrm{Ka}$ cal BC. Gnawing by a medium-sized carnivore, either a wild species or dog, was observed on two caprine bones from Sector B (A/ C-deposits). With the exception of one goat scapula from the A-deposits, none of the caprine bones could be identified to species level (Fig. 20) (cf. (Boessneck et al. 1964)). Remains identified as small bovid, mainly tooth fragments, were not diagnostic enough to allow a distinction to be made between domestic and wild, small bovid species. In some cases, gazelle, which is the most slender of the small bovid species, could be excluded, resulting in identification as caprine, ibex or Barbary sheep. A category of unidentified large bovids was also distinguished. The few tooth splinters and the humerus proximal epiphysis fragment attributed to this category could represent either a wild species, for 


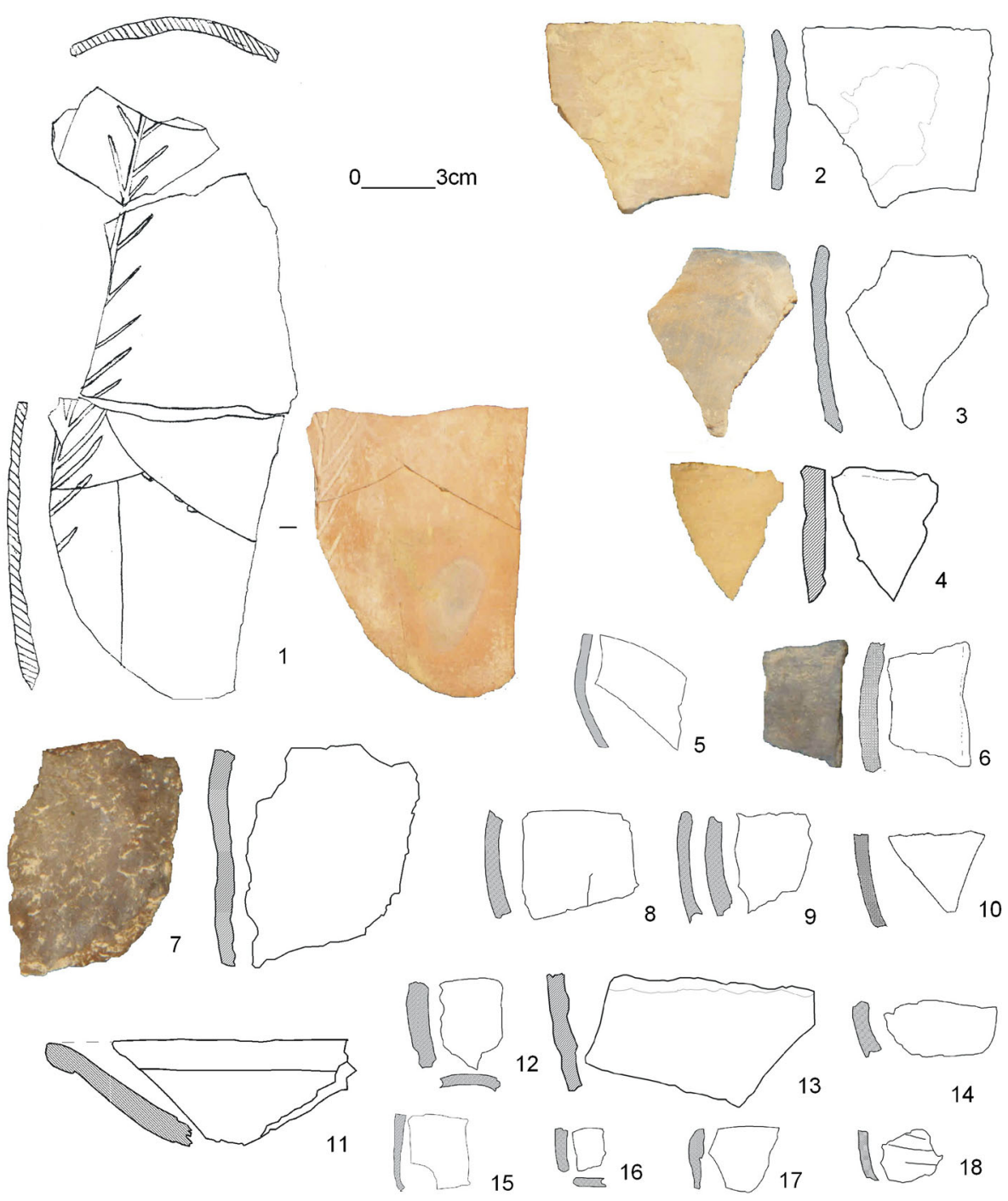

Fig. 19 Ceramics

example addax (Addax nasomaculatus), oryx (Oryx dammah) or domesticated cattle (Bos primigenius $\mathrm{f}$. taurus).

All the animals recorded still survive in the area today (Osborn and Helmy 1980), and no significant environmental changes therefore need to be supposed. Rock dassie has now disappeared from the region as a result of extensive cutting of acacia trees (Osborn and Helmy 1980, p. 465). There are no obvious changes in the faunal composition from the oldest Holocene horizons to the younger ones

Organic deposits, mainly composed of dung, are especially extensive in the Ahorizon, with an accumulation in the gully depression up to $50 \mathrm{~cm}$ thick, over a surface of at least $50 \mathrm{~m}^{2}$. Several combined arguments suggest that the dung deposits were produced by domesticated caprines: the size and weight of the individual pellets, the 
Table 6 Faunal remains recovered from the Holocene deposits at Sodmein, excepting dung pellets

\begin{tabular}{|c|c|c|c|c|c|}
\hline & & A-B & $\mathrm{C} 1$ & $\mathrm{C} 2$ & Total \\
\hline Landsnails & Zootecus insularis & 0 & 32 & 0 & 32 \\
\hline \multirow[t]{2}{*}{ Red Sea shells } & Dentalium cf. reevei & 0 & 1 & 0 & 1 \\
\hline & Cowry (Cypraeidae) & 0 & 1 & 0 & 1 \\
\hline Amphibians & Frog or toad & 0 & 2 & 0 & 2 \\
\hline \multirow[t]{2}{*}{ Reptiles } & Small lizard (Agamidae?)-MNI & 1 & 11 & 1 & 13 \\
\hline & Small snake & 0 & 2 & 0 & 2 \\
\hline \multirow[t]{6}{*}{ Birds } & Dove (Streptopelia sp.) & 0 & 1 & 0 & 1 \\
\hline & Swift (Apus apus or Apus pallidus) & 0 & 6 & 0 & 6 \\
\hline & Small Passeriformes & 0 & 1 & 0 & 1 \\
\hline & Unidentified bird & 0 & 11 & 1 & 12 \\
\hline & Ostrich (Struthio camelus) eggshell & 1 & 1 & 0 & 2 \\
\hline & Pellet bird of prey & 1 & 0 & $1^{\mathrm{a}}$ & 2 \\
\hline \multirow[t]{16}{*}{ Mammals } & Hare (Lepus capensis) & 0 & 0 & 1 & 1 \\
\hline & Microbats (Microchiroptera) & 1 & 5 & 0 & 6 \\
\hline & Shrew (Crocidura sp.) & 1 & 0 & 0 & 1 \\
\hline & Small rodent-MNI & 7 & 30 & 65 & 43 \\
\hline & & Of which Jird (Meriones sp.) & 1 & 0 & 0 \\
\hline & & $\begin{array}{l}\text { Of which Gerbil (Gerbillus } \\
\text { spp.) (min. } 3 \text { species) }\end{array}$ & 3 & 6 & 3 \\
\hline & Rock dassie (Procavia capensis) & 6 & 5 & 5 & 16 \\
\hline & Cat (Felis sp.) & 0 & 1 & 0 & 1 \\
\hline & Rüppell's fox (Vulpes rueppellii)-MNI & 0 & 3 & 0 & 3 \\
\hline & Dorcas gazelle (Gazella dorcas) & 0 & 7 & 1 & 8 \\
\hline & Goat (Capra aegagrus f. hircus) & 1 & 0 & 0 & 1 \\
\hline & Sheep (Ovis ammon f. aries) or goat & 2 & 3 & 4 & 9 \\
\hline & $\begin{array}{l}\text { Sheep, goat, Barbary sheep (Ammotragus } \\
\text { lervia) or ibex (Capra ibex) }\end{array}$ & 2 & 7 & 3 & 12 \\
\hline & Small bovid & 4 & 102 & 15 & 121 \\
\hline & Large bovid & 0 & 7 & 1 & 8 \\
\hline & Unidentified & 126 & 586 & 46 & 758 \\
\hline Grand total & & 157 & 831 & 87 & 1063 \\
\hline
\end{tabular}

Unless otherwise indicated, the figures are numbers of identified specimens (NISP). It should be noted that compared to the preliminary species list (Linseele et al. 2010), additional samples have been included

$M N I$ minimum number of individuals

${ }^{\text {a }}$ Containing remains of a minimum of two small rodent individuals

large quantities deposited and the presence of hearths and artefacts among the deposits, indicating animals under human management, and the species present among the Holocene bone remains (Linseele et al. 2010). Caprines were presumably penned in the cave, a practice that is attested both archaeologically and ethnographically elsewhere (see references in Linseele et al. 2010). However, Sodmein Cave is the first archaeological example from the Egyptian Eastern Desert. 

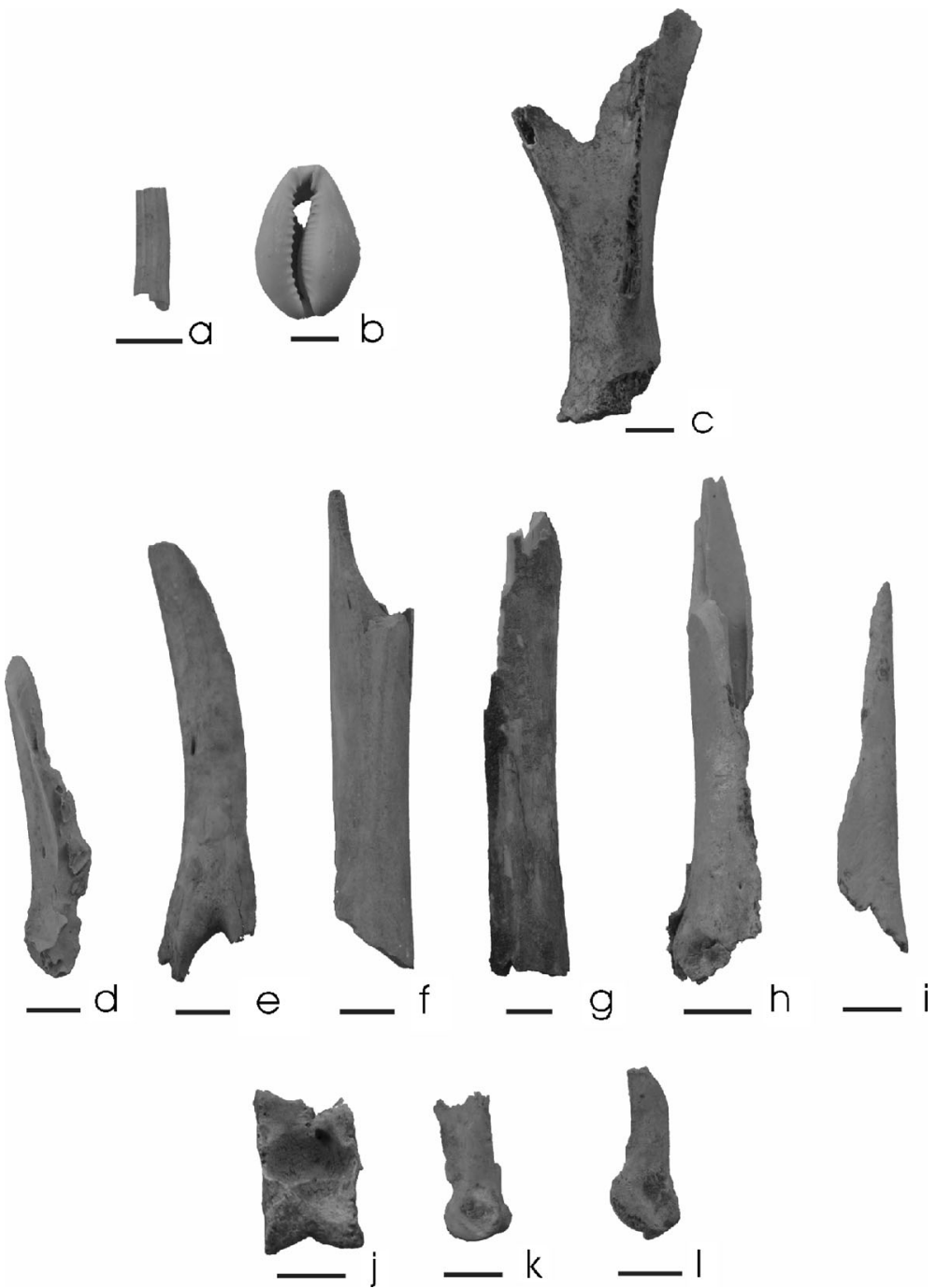

Fig. 20 Red Sea shells and bones identified as caprine from Holocene levels at Sodmein. a Dentalium cf. reevei; b cowry_ventral view; c goat scapula-lateral view; d caprine scapula-lateral view; e caprine humerus diaphysis - caudal view; f caprine femur diaphysis - caudal view; g caprine tibia diaphysis - plantar view; h caprine metatarsal - dorsal view; i caprine metatarsal — dorsal view; j caprine talus, dorsal view; k caprine phalanx 1 -side view; I caprine phalanx 1 - side view. Scale bar $=1 \mathrm{~cm}$

\section{Plant Remains}

Wood identifications were conducted on wood charcoal samples, mainly from hearth features (Table 2). For the analysis, all available wood charcoals bigger than $0.5 \mathrm{~cm}$ were selected and studied under a binocular microscope. Subsequently, identifications were made by production of fresh fractures on three surfaces (transversal, radial and tangential) and observing them under a reflected light microscope. In total, 1409 wood charcoal fragments were analysed, of which 892 proved identifiable. Almost $60 \%$ of the wood available for analysis from the $\mathrm{C} 2$-deposits was unidentifiable, because of 
strong alteration of its morphological structure (resembling "melting"). The study identified the highest diversity of wood charcoal taxa in the C1-deposits (Fig. 21). The wood assemblages from the C2-deposits contain the same dominant taxa (Acacia, Tamarix, Salvadora persica) as those of the C1-deposits. However, the fact that no other taxa are present is most probably due to poor preservation of the charcoals in this horizon.

Further information on the past vegetation is available from archaeobotanical analyses of dung pellets from Sodmein (Table 7). From the Holocene layers A and $\mathrm{C}$, a total of 17 dung pellets have been analysed macrobotanically (Linseele et al. 2010). The methodology of Kühn and Hadorn (2004) was followed, with slight modifications. No pollen was found preserved and therefore no further palynological samples were prepared for study.

The plant macrofossils recognised in the pellets consist mainly of small seeds and wood material (Table 7). It should be noted that all of the identified plant taxa correspond to the natural vegetation and that cultivated crops are completely absent. Some larger fragments of wood could be attributed to Fabaceae, in two cases these most probably belong to acacia ( $c f$. Acacia). In addition, seed fragments of Acacia were found. Most of the small seeds collected from the dung could be identified as Poaceae (including some Panicoideae) and Asteraceae (including cf. Pulicaria sp. and Artemisia sp.). A couple of seeds most probably belonging to salt-tolerant Aizoon canariensis were also present. In a few cases, small leguminous seeds (like cf. Lotus sp.) also appeared in the studied material. Other plant remains that could be recognised are leaf epidermis, leaf fragments, charcoals and fragments of seed testa/fruit coat. The leaves, wood fragments and the seeds of Acacia found in the pellets indicate browsing of the tree vegetation by the bovids that produced the dung. As wood charcoal analysis of the Tree Shelter site (Marinova 2008; Marinova et al. 2008) and of Sodmein (Fig. 21) has shown, S. persica was also used by the prehistoric visitors of the area. The leaves of Salvadora also make good fodder, as

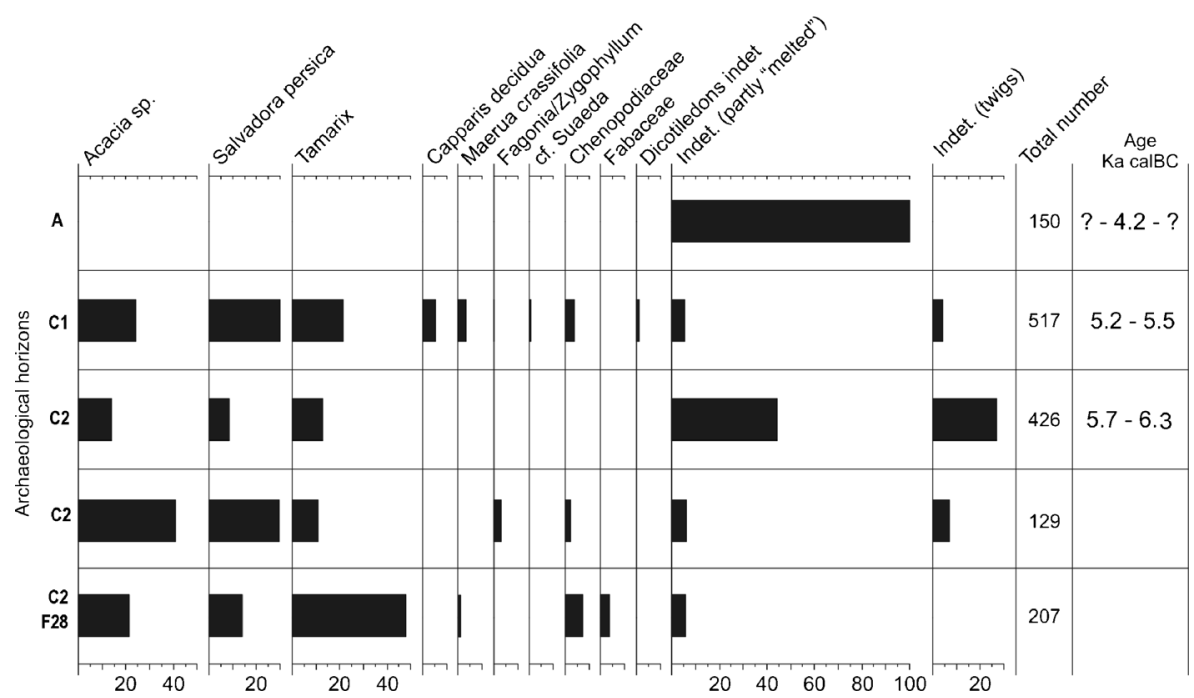

Fig. 21 Wood charcoal identified from the Holocene levels (mainly from hearth contexts—see Table 1) presented as percentage 
Table 7 Results of the macrobotanical analysis of dung pellets from the Sodmein Cave summarised for the Holocene layers (+: fewer than 10 items, ++: between 10 and 30 items, +++: more than 30 items)

\begin{tabular}{|c|c|c|}
\hline Archaeological horizon & A-B & $\mathrm{C}$ \\
\hline Number of studied coprolites & 12 & 5 \\
\hline Acacia (seed) & 1 & - \\
\hline Acacia (leaf) & 4 & 2 \\
\hline cf. Acacia (wood) & - & - \\
\hline Fabaceae (wood) & - & 1 \\
\hline Aizoon cf. canariense (seed) & 1 & 1 \\
\hline Artemisia sp. (achena) & 1 & 4 \\
\hline cf. Pulicaria (achene) & 10 & 4 \\
\hline Asteraceae (achene) & 2 & 3 \\
\hline$c f$. Geraniaceae (fruit) & 8 & 5 \\
\hline Fabaceae (seed) & 2 & - \\
\hline cf. Lotus (seed) & - & 1 \\
\hline Panicoideae (caryopsis) & 3 & 1 \\
\hline cf. Eragrostis/Sporolobus (caryopsis) & 18 & 10 \\
\hline cf. Pennisetum (caryopsis) & 1 & 2 \\
\hline Poaceae (caryopsis) & 15 & 3 \\
\hline cf. Plantago/Veronica (seed) & 2 & - \\
\hline Small seed/fruit indet. & 9 & 5 \\
\hline Fruit/seed fragment indet. & + & - \\
\hline Leaf nerve & + & + \\
\hline Wood fragment indet. & ++ & ++1 \\
\hline Poaceae stem fragment & +++ & ++ \\
\hline Charcoal fragment indet. & + & - \\
\hline
\end{tabular}

their water content is high (15-36\%) (Von Maydell 1986). Since it was not possible at the time to have a palaeobotonist working at the site during excavation, apart from the charcoals and the macroremains inside the pellets, no plant remains have been collected.

\section{Sodmein in a Wider Context}

Assemblages of a similar date from the Eastern Desert or from the Nile Valley are almost entirely absent. Nevertheless, some groups in and outside the Nile Valley bear some resemblances with the Sodmein material (Fig. 22).

\section{Eastern Desert}

Montenat (1986) observed, after a survey of the Red Sea coast, that Neolithic sites are almost entirely absent. Our knowledge of what happened during the Early and Middle Holocene is thus very restricted. 


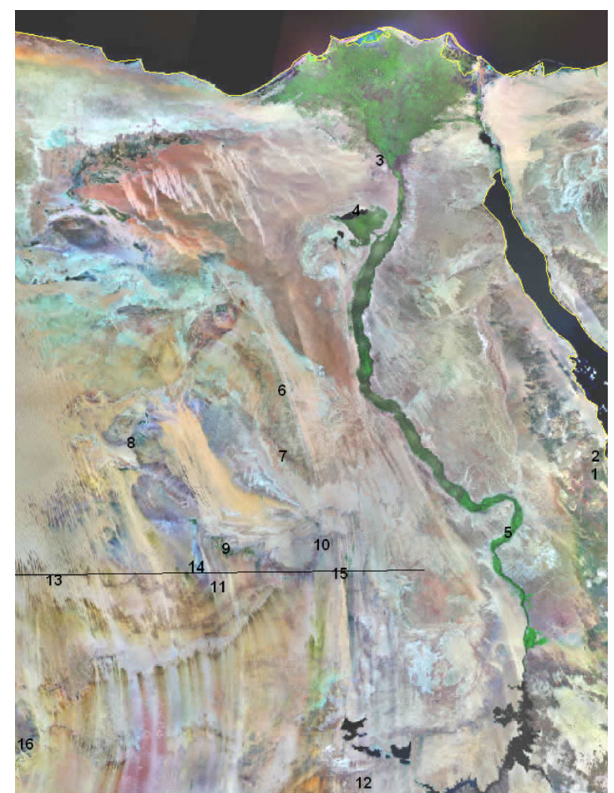

Fig. 22 Early and Middle Holocene sites. Satellite picture (NASA Egypt_A2004201_0830_250m.jpg) of Egypt with sites mentioned in the text: 1: Sodmein Cave, 2: Tree Shelter, 3: Merimde, 4: Fayum, 5: El Tarif, 6: Djara, 7: Abu Gerara, 8: Farafra, 9: Dakhla, 10: Abu Tartur, 11: Eastpans, 12: El Ghanam, 13: Regenfeld, 14: Chufu, 15: Kharga, 16: El Gouna. Horizontal line separates the northern from the southern area of the Western Desert

Several Holocene occupation horizons have been defined at the Tree Shelter site (Vermeersch 2008) within a time period between 7.3 and 3.7 Ka cal BC. The Tree Shelter site is situated some $3 \mathrm{~km}$ to the north of Sodmein Cave. The Epipalaeolithic Elkabian at Tree Shelter was well represented in horizon AH5 and had several small hearths dating from 7.1 to $6.5 \mathrm{Ka}$ cal BC. This broadly coincides with the earliest Holocene Sodmein occupation (feature 310), which is nevertheless of an entirely different type from the Tree Shelter site features. The elongated Ounan points (Fig. 15: 4-5) recovered from the Sodmein deposits were apparently not fabricated using the microburin technique, and thus cannot be considered to be Abu Tartur points (Riemer et al. 2004). They have been ${ }^{14} \mathrm{C}$-dated to about $6.5 \mathrm{cal} \mathrm{BC}$.

The lithics of AH3 at the Tree Shelter site (Vermeersch 2008), with ages from 5.7 to 4.7 Ka cal BC, are similar to those of the Sodmein C-horizon. Save for a side-blow flake, there are no major differences with Sodmein. The fauna from the Tree Shelter site is very comparable to that of the Sodmein Cave. However, microbats, typical cave fauna, are missing and remains of marine fish have been identified, whereas no fish were recorded from Holocene Sodmein (Linseele and Van Neer 2008; Marinova et al. 2008).

At Wadi Araba, near Bir Buerat, arrowheads, suggesting a PPNB connection, have been reported (Tristant 2010). They are similar to those from Sodmein. At Gebel Zeit (Dittmann 1990), several elongated leaf-shaped arrowheads and a stemmed arrowhead, both with bifacial retouch, similar to those from Sodmein (Figs. 15, 16-18), were found at site B. Our survey in Wadi Bili (Vermeersch et al. 2005a) found a small huntergatherer site dated to $4.7 \mathrm{Ka}$ cal BC (GrA-23727) and a shell mound at El Gouna of 
similar age. The archaeological material is, however, very limited. The Tasian material from Wadi Atulla (Friedman and Hobbs 2002) is dated to $4.7 \mathrm{Ka}$ cal BC.

\section{Nile Valley}

Merimde Benisalâme $\mathrm{I}$ is situated on the western edge of the Nile delta, and the Urschicht (Eiwanger 1984) is dated about 4.7-4.5 Ka cal BC, somewhat younger than the main occupation of Holocene Sodmein. The general structure of debitage products and tools is quite similar and simple: cores are mainly single platform cores, of which most are exhausted. Among the retouched artefacts, some bifacial tools are present. Some are borers and arrowheads (Eiwanger 1979), but large bifacial leaf-shaped artefacts, similar to those from Sodmein, are also present. The Urschicht ceramics are mostly thin-walled and characterised by an untempered fabric. Typical of this Urschicht pottery is a form of herringbone decoration, incised before firing, on some of the polished pottery. This decoration is similar to that of a decorated pot from Sodmein (Fig. 19: 1), although this is probably much older (6.5 Ka cal BC).

With an age around 4.5 Ka cal BC, the Fayumian (Wendrich et al. 2010; Shirai 2010 ) is younger than Sodmein. The Moerian is younger still.

The Tarifian at El Tarif (Ginter et al. 1979) in Upper Egypt is not well dated, but is seemingly older than the overlying Predynastic. A single date of $5.3 \mathrm{Ka}$ cal BC (Gd1756) is available from site MA 2/83 near Armant (Ginter and Kozlowski 1994). The knapping technique is simple, often without any preparation of the core. The main category of tool is the retouched flake. Side-scrapers are the second most characteristic lithic artefact. Twelve retouched bifacial tools can probably be interpreted as axes. It is significant that notches and denticulates are absent from the Tarifian record. The pottery fabric is primarily tempered with plant material. There is very little resemblance between the Tarifian and the Sodmein assemblages.

Although partially contemporaneous, the Shamarkian (Schild et al. 1968) is quite different from the Sodmein material. Although the Egyptian Predynastic is later than the Sodmein assemblages, its lithic industry is primarily flake-based with some bifacial tools and, as such, is similar to Sodmein.

\section{Western Desert}

In the Western Desert, numerous Early and Middle Holocene sites have been excavated and dated (Wendorf et al. 2001; Gehlen et al. 2002; McDonald 2003). Many sites, belonging to the Egyptian Western Desert bifacial technocomplex, Flächenretuschiertes Komplex, present similarities with the Sodmein artefacts from the C-deposits. These similarities are best understood in relation to the Djara group. Djara is situated in the Western Desert on the Abu Muhariq Plateau at the latitude of Asyut (Kindermann 2010). The lithic material is characterised by opportunistic flaking, producing mainly flakes from single platform cores and is thus similar to the Sodmein debitage techniques. Tool types are rather similar to those from Sodmein and clearly belong to the bifacial technocomplex of the Western Desert. Kindermann (2010) stresses that it is rather difficult at Djara to attribute a specific arrowhead type to a specific time period since many such pieces are surface finds. The arrowheads from Djara 90/1, cluster 7, corresponding to Djara B with an AMS date of $5.9 \mathrm{Ka}$ cal BC 
(KIA-20681), are quite comparable to those from the Sodmein cave. However, facially retouched projectile points appeared for the first time in Djara A. The earliest radiocarbon date of $6.4 \mathrm{Ka}$ cal $\mathrm{BC}(\mathrm{KN}-4602)$ on the Abu Muhariq Plateau for facially retouched points comes from site Djara 90/1-2. Although bifacially retouched points occur during Djara A, the most characteristic forms are laterally retouched stemmed or lens-shaped points. Points with facial retouch are also known from the Bashendi A unit of Dakhla Oasis (McDonald 1992). However, a Masara C site, Loc 265, dating somewhat later than most Masara C sites, yielded tanged and leaf-shaped points, some with bifacial edge retouch (McDonald 2003). Tranchet axes, represented at Sodmein by a tranchet-preparation flake from $\mathrm{C} 1$, seem to occur exclusively as a tool type during Late Djara B (Kindermann 2010). Side-blow flakes, regarded as characteristic of Late Djara B, but already present during Early Djara B, are lacking at Sodmein but were present at the Tree Shelter site in AH3 (5.5-5.3 Ka cal BC) (Vermeersch 2008). No grinding implements were found at Sodmein and the Tree Shelter site, whereas in the Western Desert they are clearly attested from Djara A onwards (Gehlen et al. 2002).

Pottery does not occur at Djara A sites, apart from a few thin-walled shards, which might have parallels to fabrics from the Late Bashendi A in Dakhla (Hope 2002; Kindermann 2004; Riemer and Schönfeld 2010). It seems that the Djara B pottery fits best with the majority of the Sodmein pottery. According to Gehlen et al. (2002), there is a clear typological similarity between Djara B and the late Bashendi A (6.45.7 Ka cal BC) at Dakhla Oasis (McDonald 1991, 1992) and also with the Neolithic assemblages of Kharga (Caton-Thompson 1952) and Farafra (Barich and Hassan 1987). Such sites from the Egyptian Western Desert, belonging to the bifacial technocomplex, also present similarities with Sodmein. Shells of the Nile bivalve Aspatharia sp. (also called Spathopsis sp. or Chambardia) provide evidence for contacts between Djara and the Nile Valley (Kindermann and Bubenzer 2007). The presence of a pair of cowry shells at site 270 (McDonald 2013) suggests contact also with the Red Sea area.

The sites from Regenfeld, Abu Jerar, Mudpans, Gilf, Nabta Playa and the sites occupied by the Ruat el Ghanam mixed pastoralists, which lie south of a line at latitude $25.5^{\circ} \mathrm{N}$ (Fig. 24), clearly belong to another facies characterised by transverse arrowheads and other geometries.

Domesticated caprines probably appear slightly later in the Western than in the Eastern Desert and have been found at several sites dated to the sixth millennium BC (Gautier 1984, 2014; McDonald 1991; Churcher et al. 2008; Pöllath 2010). In contrast to older sites in the Western Desert, large faunal samples have been recorded for KS043 in the Kharga Oasis (4.8-4.4 Ka cal BC) (Lesur et al. 2011). These are mainly composed of domesticates, which, in order of decreasing importance, comprise sheep, goat and cattle.

\section{Palaeoenvironmental Conditions}

The cave deposits are thought to have remained under very dry conditions since their sedimentation (Moeyersons et al. 2002). 
The Early Holocene seems to be a period when a wetter climate is most likely. In the Eastern Sahara, at this latitude, it seems that the wettest period was around $7.0 \mathrm{Ka}$ cal BC (Moeyersons et al. 1999; Wendorf et al. 2001; Kuper and Kröpelin 2006). At the Tree Shelter site, humans (Elkabian) exploited the desert around $7.0 \mathrm{Ka}$ cal BC (Vermeersch 2008) and yet by $5.7 \mathrm{Ka}$ cal BC the Elkabian deposit (AH5) had been partially eroded by wadi activity. At Sodmein, the anomalous absence of Elkabian may be explained by the likelihood that, posterior to the Pleistocene deposits, an erosive phase created the depression filled with the main archaeological zone deposits. Despite this erosion, an Elkabian human presence is attested by the presence of some bladelets and backed bladelets of a typical Elkabian type (Vermeersch 1978) and by the age of feature 310. The erosional event created the base of the present day Holocene deposits. The erosional event may be dated between 7.0 and $6.2 \mathrm{Ka}$ cal BC.

The final part of this period coincides with a drought event recorded at Lake Tana and other East African lakes (Marshall et al. 2011). The period between 6.5 and $6.2 \mathrm{Ka}$ cal BC seems to coincide with the cold and dry $8.2 \mathrm{Ka}$ event (ca. $6.3 \mathrm{Ka}$ cal BC) known from the Greenland ice cores (Thomas et al. 2007), which is most probably pancontemporaneous with the dry event of the Post-Al Jerar Arid Phase (Schild and Wendorf 2013). The Holocene 8.2 Ka event in the Egyptian Eastern Desert was apparently too dry and inhospitable for humans.

Starting from 6.2 Ka cal BC, an important accumulation of mass movement (Cdeposits) with a high content of organic material was created. Many successive visits by humans are attested by the lithics, ceramics, faunal remains and excavated features.

After 5.3 Ka cal BC, the gully depression was created due to an exceptional erosional event. The fill of the gully (A- and B-deposits) is mainly characterised by an accumulation of caprine dung, suggesting use of the cave for the stabling of domestic animals. Apparently, these people did not introduce any other significant archaeological remains. There seems to be another kind of utilisation of the cave before and after the $5.3 \mathrm{Ka}$ cal $\mathrm{BC}$ period. Or, more probably, there were far fewer people visiting the area. In the filling deposits, hearths are clearly less numerous. As soon as it became very dry, after $4.2 \mathrm{Ka}$ cal $\mathrm{BC}$, no humans used the cave; indeed it would seem that very few people ventured into the desert. At the best of times, the valleys of the Eastern Desert were a low, thorn-tree and sparse grass savannah landscape of semidesert type: much better than the degraded, acacia palimpsests of today, but hardly a habitat for large herds or plentiful pastoralists. Full, contemporary aridity was established in the Eastern Desert by 3.6 Ka cal BC (Wilkinson et al. 2004).

Between approximately 7.5 and 6.1 Ka cal BC, the northern Red Sea Mountains registered substantially higher rainfall and freshwater runoff, which thereafter decreased to modern values (Arz et al. 2003). In an earlier stage in the study of Holocene climatic phases in the Eastern Sahara, Barich and Hassan (1987) suggested a succession of several wet and dry phases. However, the radiocarbon dates from that area do not indicate any rupture in regional climatic development between 7.0 and $5.3 \mathrm{Ka}$ cal BC (Kuper and Kröpelin 2006). Nevertheless, it is beyond discussion that an important cultural change occurred between the Epipalaeolithic and later occupation of the Eastern Desert, with an absence of visitors during the Holocene cold and dry $8.2 \mathrm{Ka}$ event. The question of whether some cultural changes may have taken place for reasons related to that climatic event cannot yet be resolved. 


\section{Economy}

Microwear analysis on stone tools from the Elkabian of the Tree Shelter site indicates mainly hide working and hunting activities (Kweakason 2008). The presence of domestic sheep or goat in the Elkabian levels of Tree Shelter is very unlikely (Linseele and Van Neer 2008; Marinova et al. 2008). Sodmein Cave does not provide more information regarding the economic activity of the Elkabian visitors. The economy of such visitors probably consisted solely of hunting and gathering.

The occupants of the C-deposits from Sodmein and of the Tree Shelter AH2 and AH3 horizons might be characterised as mobile livestock keepers. As such, they can be considered as Neolithic (Linseele and Van Neer 2008). These groups engaged in animal husbandry, and no indications of agriculture are present. Admittedly, the collection of archaeobotanical data was not ideal, but considering the archaeobotanical record from contemporary sites ( $c a .7$ to $4 \mathrm{Ka}$ cal BC; see Fahmy 2001; Wasylikowa 2001; Thanheiser 2011), no evidence for agriculture or agricultural products is to be expected. Even grinding stones, present at Bashendi (McDonald 2013) and Djara sites (Kindermann 2010), are absent. Could this be related to an absence of grain collecting?

In general, there is very little evidence for meat consumption at the sites, whether of wild or domesticated animals. Only 10 bones could be attributed to domesticated caprines. The large size of the dung accumulations in the A and B-horizon suggests that the size of the herds was larger than would be suspected from the scant bone remains found. Nevertheless, very large herds need not be presumed, as repeated visits after $5.0 \mathrm{Ka}$ cal $\mathrm{BC}$ could also have produced the observed accumulations. The use of ephemeral vegetation for the livestock herds was probably the main reason for visiting the Sodmein Cave. Both Sodmein and Tree Shelter were presumably used repeatedly, but for short periods only, as places where livestock was temporarily sheltered. The lack of human food remains may be largely due to the ephemeral nature of the occupation. In addition, the livestock could have been kept for milk, rather than for meat. As has been shown for Eurasia (Vigne and Helmer 2007), milk may have had an important economic role already at the beginning of the Neolithic. Its extensive use has been proven for the fifth millennium BC in Libya (Dunne et al. 2012). Does the presence of arrowheads point to the importance of hunting in the Neolithic?

Despite the scarcity of bone remains, the presence of domestic caprines at Sodmein Cave is of great importance for the early history of the dispersal of these animals in the African continent. The remains are present from the oldest Holocene deposits onwards (i.e., the $\mathrm{C} 2$-deposits). This indicates that caprines were present from about $6.2 \mathrm{Ka}$ cal $\mathrm{BC}$ in the area, from at least $6.2 \mathrm{Ka}$ cal BC, possibly earlier than in the Western Desert. The animals were domesticated in an area encompassing the Northern Levant and the Zagros Mountains (Muigai and Hanotte 2013) and spread from there. Because of the very limited bone evidence for early caprines in northeastern Africa, a reconstruction of their routes of dispersal must remain very tentative, with various possible dispersal routes, from the Western Desert or over the Red Sea Coast, or even via the Nile Valley to the Sodmein area (Riemer and Kindermann 2007). It is assumed here that sheep and goat dispersed together, but it should be emphasised that goat is the only one of the two represented by diagnostic remains at both Sodmein and the Tree Shelter site (Linseele and Van Neer 2008, Linseele et al. 2010). 


\section{Conclusions}

The Holocene prehistoric remains are attributed to hunter-gatherers of the Elkabian and for the later occupants to groups of herders. The presence of numerous arrowheads could suggest that the herders were also hunters, but this cannot unequivocally be confirmed through the faunal remains. When analysing the series of ${ }^{14} \mathrm{C}$ dates from Sodmein and the Tree Shelter site, several periods of Holocene geomorphologic events and human occupation phases can be defined (Figs. 23 and 24) from early to late:

1. An Elkabian occupation phase around 7.1 Ka cal BC which left several occupation horizons at the Tree Shelter site (Vermeersch 2008) but from which at Sodmein only a single feature (F310) was preserved together with some scattered artefacts. At the Tree Shelter site, it continues to be present up to $6.6 \mathrm{Ka}$ cal BC. Gehlen et al. (2002) have observed that mid-Holocene occupations of the Western Desert evidence a strictly flake-orientated tool production, which replaced the elaborate blade and bladelet technology of the Epipalaeolithic. This replacement, attested at Djara at $6.4 \mathrm{Ka}$ cal BC (Kindermann 2010), is clearly present at the Tree Shelter site and Sodmein Cave only from a somewhat later time period $(6.3 \mathrm{Ka}$ cal BC).

2. An erosional event occurred, presumably between 6.5 and $6.2 \mathrm{Ka}$ cal $\mathrm{BC}$. This time period coincides also with a drought event in the upper reaches of the Nile (Marshall et al. 2011). The period between 6.5 and $6.2 \mathrm{ka}$ cal $\mathrm{BC}$ seems also to coincide with the cold and dry 8.2 Ka event ( $c a .6 .3 \mathrm{Ka}$ cal BC) known from the Greenland ice cores (Thomas et al. 2007). That Holocene $8.2 \mathrm{ka}$ event in the Egyptian Eastern Desert was apparently too dry and inhospitable for humans.

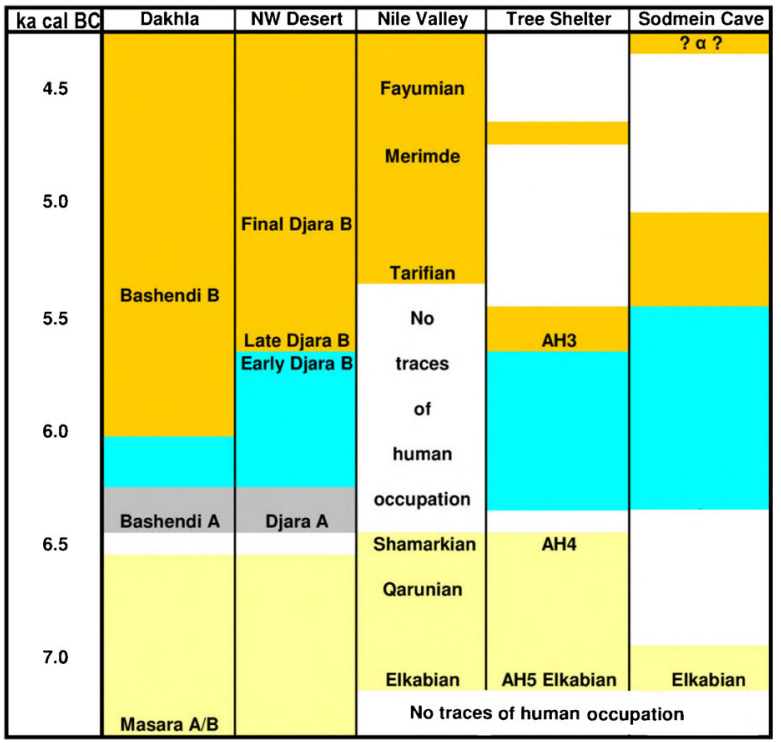

Fig. 23 Chronological position of Holocene sites in Egypt. Chronological $\left({ }^{14} \mathrm{C}\right)$ position of different Holocene archaeological units in Egypt. Yellow: Epipalaeolithic, Grey: start of the Egyptian bifacial technology, Blue: start of herding, Orange: presence of ceramics (image in full colour online) 


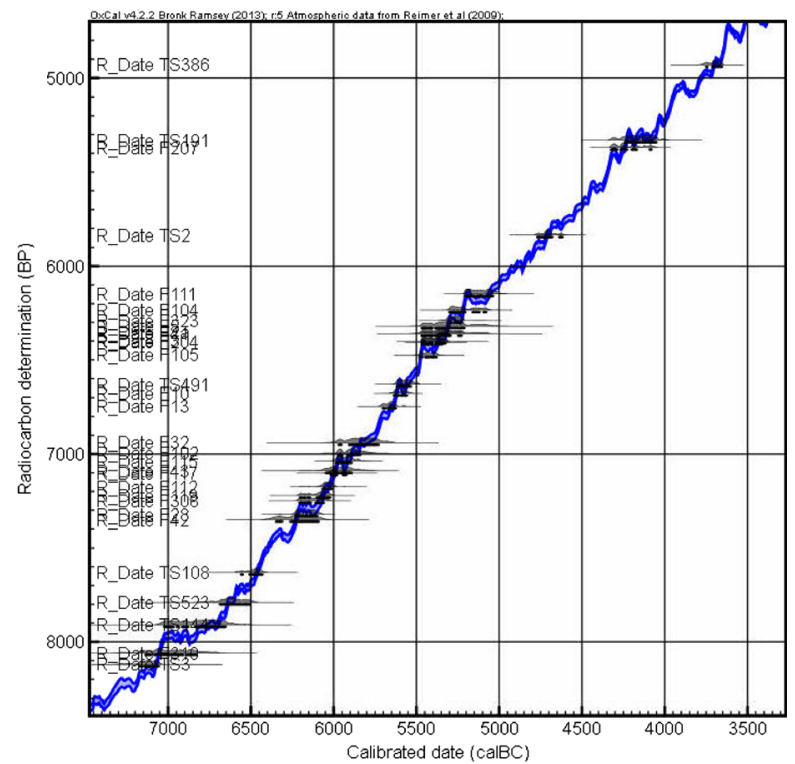

Fig. 24 Calibration of the Sodmein $(F)$ and Tree Shelter site $(T S){ }^{14} \mathrm{C}$ dates using OxCal v4.2.2 (Bronk Ramsey 2009)

3. An occupation by mobile stock-keepers with caprines is mainly centred on a time period of 6.2-5.8 Ka cal BC. They used lithics, which fit within the north-western bifacial technocomplex, and they probably did not use ceramics. Caprine excrement is always present but did not build up thick accumulations.

4. A period of some centuries (5.8-5.4 Ka cal BC) seemingly coincides with reduced visitation of the site, as far as may be supposed from the reduced number of ${ }^{14} \mathrm{C}$ dates available.

5. A renewed occupation between 5.4 and $5.0 \mathrm{Ka}$ cal $\mathrm{BC}$ by herders with caprines, lithics of the same north-western bifacial technocomplex, but this time apparently with some ceramics.

6. An important erosion event posterior to $5.1 \mathrm{Ka}$ cal BC, creating the gully depression (Fig. 9) in Sodmein Cave.

7. New groups of herders visited the cave. They sheltered herds of caprines in the cave, depositing a thick layer of excrement in the gully depression at Sodmein. The exact time period and the duration of this fill is unknown but F207, with an age of about $4.3 \mathrm{Ka}$ cal $\mathrm{BC}$, suggests a time period that could coincide with the ${ }^{14} \mathrm{C}$ dates at the Tree Shelter site at 4.3 and 3.7 Ka cal BC (Vermeersch 2008).

8. After those later visits, the caves remained deserted for a very long time period.

In many regions of the Western Desert, there is a clear drop-off in the number of ${ }^{14} \mathrm{C}$ dates just after 5.4/5.3 Ka cal BC. This reflects the beginning of a trend towards hyperarid conditions, which led to a depopulation of the desert after 5.0 cal BC (Kuper and Kröpelin 2006). In the Eastern Desert, desertification seems to occur somewhat later. This situation is probably related to the presence of the Red Sea Mountains where orographic rainfall might have been frequent. 
A fundamental change in technology and blank procurement for tool production, visible in the transition from the Epipalaeolithic to Djara A around 6.5 Ka cal BC, is also observed in the Red Sea area, where the presence of the new technology is attested at all younger sites, such as Sodmein Cave, the Tree Shelter site (Vermeersch 2008), the Wadi Bili Neolithic site (Vermeersch et al. 2005a) and the El Gouna shell mound (Vermeersch et al. 2005b). Is there a connection between this change in technology and the cold and dry Holocene $8.2 \mathrm{Ka}$ event?

In the Western Desert, cultural traditions are divided by a north-south, rather than a west-east change in material diversity (Fig. 22). The Egyptian North-Western Desert and Eastern Desert share similar cultural traditions. The basic motor behind this cultural spread may have been the climatic gradient between the northern and southern Sahara, primarily differences in the distribution of summer-winter rain.

Acknowledgments The Netherlands-Flemish Institute in Cairo took care of all administrative duties in Egypt and provided us with housing while in Cairo.

We have always enjoyed good collaboration with the representatives of the Supreme Council of Antiquities. They truly facilitated the administration in Cairo, permitting us to be in the field within just a few days after our arrival in Egypt. We could rely on the Qena inspectorate for assistance, and the inspectors assigned to our team were very helpful. They co-ordinated our contacts with the local population and solved all kinds of problems that so often occur during excavations. Special thanks go to M. Hussein Ahmed Hussein El Afiuni, chief inspector at Qena, who became a good friend during the many years we worked in the area. The motivation and good temper of the local workmen were a relief in the sometimes harsh desert work environment.

We thank many students who were very helpful in the field and in analysing the archaeological materials. The authors are moreover indebted to M. Van Meenen for artefact drawings, and to J. Meersmans for taking care of our equipment. The contribution of Wim Van Neer to this paper presents research results of the Interuniversity Attraction Poles Programme-Belgian Science Policy. Veerle Linseele is a postdoctoral research fellow of the FWO-Flanders. A final word of thanks goes to all our helpful colleagues, especially to Dr. P. Van Peer and Dr. E. Paulissen, for field work and critical discussions, and to the two anonymous reviewers who gave us some very good advice.

Open Access This article is distributed under the terms of the Creative Commons Attribution 4.0 International License (http://creativecommons.org/licenses/by/4.0/), which permits unrestricted use, distribution, and reproduction in any medium, provided you give appropriate credit to the original author(s) and the source, provide a link to the Creative Commons license, and indicate if changes were made.

\section{References}

Arz, H. W., Lamy, F., Pätzold, J., Müller, P. J., \& Prins, M. (2003). Mediterranean moisture source for an earlyHolocene humid period in the northern Red Sea. Science, 300, 118-122.

Barich, B. E., \& Hassan, F. A. (1987). The Farafra Oasis Archaeological Project (Western Desert, Egypt). Origini, 13, 117-191.

Boessneck, J., Müller, H., \& Teichert, M. (1964). Osteologische Unterscheidungsmerkmale zwischen Schafe (Ovis aries Linne) und Ziege (Capra hircus Linne). Kühn-Archiv, 78, 1-129.

Bronk Ramsey, C. (2009). Bayesian analysis of radiocarbon dates. Radiocarbon, 51, 337-360.

Bubenzer, O., Bolten, A., \& Darius, F. (Eds.). (2007). Atlas of cultural and environmental change in arid Africa. Köln: Heirich-Barth-Institut.

Caton-Thompson, G. (1952). Kharga Oasis in prehistory. London: Royal Anthropological Institute.

Churcher, C. S., Kleindienst, M. R., Wiseman, M. F., \& McDonald, M. M. A. (2008). The Quaternary faunas of Dakhleh Oasis, Western Desert of Egypt. In M. F. Wiseman (Ed.), The Oasis papers 2, Proceedings of the Second International Conference of the Dakhleh Oasis Project (pp. 1-24). Oxford: Oxbow Books. 
Dittmann, A. (1990). Zur Paläogeographie der ägyptischen Eastern Desert. Der Aussagewert prähistorischer Besiedlungsspuren für die Rekonstruktion von Paläoklima und Reliefentwicklung. Marburg: Marburger Geografische Schriften 116.

Dunne, J., Evershed, R. P., Salque, M., Cramp, L., Bruni, S., Ryan, K., Biagetti, S., \& Di Lernia, S. (2012). First dairying in green Saharan Africa in the fifth millennium BC. Nature, 486, 390-394.

Eiwanger, J. (1979). Geschoßspitzen aus Merimde. Jahrbuch des Römisch-Germanischen Zentralmuseums, 26, 61-74.

Eiwanger, J. (1984). Merimde-benisalâme I - Die funde der urschicht. Mainz am Rhein: Verlag Philipp von Zabern.

Fahmy, A. G. (2001). Palaeoethnobotanical studies of the Neolithic settlement in Hidden Valley, Farafra Oasis, Egypt. Vegetation History and Archaeobotany, 10(4), 235-246.

Friedman, R., \& Hobbs, J. J. (2002). A Tasian' tomb in Egypt's Eastern Desert. In R. Friedman (Ed.), Egypt and Nubia: Gifts of the desert (pp. 178-191). London: The British Museum Press.

Gabler, K. O. (1985). Osteologische Unterscheidungsmerkmale am postkranialen Skelett zwischen Mähnenspringer (Ammotragus lervia), Hausschaf (Ovis aries) und Hausziege (Capra hircus). $\mathrm{PhD}$ dissertation, Munich University

Gautier, A. (1984). Archaeozoology of the Bir Kiseiba region, Eastern Sahara. In A. E. Close (Ed.), Cattlekeepers of the Eastern Sahara: The Neolithic of Bir Kiseiba (pp. 49-72). Dallas: Department of Anthropology, Institute for the Study of Earth and Man, Southern Methodist University.

Gautier, A. (2014). Animal remains from the hidden valley Neolithic site, Farafra Oasis, Egypt. In B. E. Barich, G. Lucarini, F. A. Hassan, \& A. M. Hamdan (Eds.), From lake to sand, the archaeology of Farafra Oasis, Western Desert, Egypt (pp. 369-374). Firenze: All'Insegna del Giglio.

Gehlen, B., Kindermann, K., Linstädter, J., \& Riemer, H. (2002). The Holocene occupation of the eastern Sahara: Regional chronologies and supra-regional developments in four areas of the absolute desert. In Jennerstrasse 8 (Ed.), Tides of the desert - Gezeiten der Wüste (pp. 85-116). Köln: Heinrich-Barth-Institut.

Ginter, B., \& Kozlowski, J. (1994). Predynastic settlement near Armant. Heidelberg: Heidelberger Orientverlag.

Ginter, B., Kozłowski, J. K., \& Drobniewicz, B. (1979). Silexindustrien von El Tarif: Ein Beitrag zur Entwicklung der prädynastischen Kulturen in Oberägypten. Mainz am Rhein: Archäologische Veröffentlichungen 26.

Hendrickx, S., Midant-Reynes, B., \& Van Neer, W. (2001). Mahgar Dendera 2 (Haute Égypte), un site d'occupation Badarien. Leuven: Leuven University Press.

Hope, C. A. (2002). Early and Mid-Holocene ceramics from the Dakhleh Oasis: Traditions and influences. In R. F. Friedman (Ed.), Egypt and Nubia: Gifts of the desert (pp. 178-191). London: The British Museum Press.

Kindermann, K. (2004). Djara: Excavations and surveys of the 1998-2002 seasons. Archéonil, 14, 31-50.

Kindermann, K. (2010). Djara. Zur mittelholozänen Besiedlungsgeschichte zwischen Niltal und Oasen (AbuMuharik-Plateau, Ägypten). Köln: Heinrich-Barth-Institut.

Kindermann, K., \& Bubenzer, O. (2007). Djara-humans and their environment on the Egyptian limestone plateau around 8,000 years ago. In O. Bubenzer, A. Bolten, \& F. Darius (Eds.), Atlas of cultural and environmental change in arid Africa (pp. 26-29). Köln: Heinrich-Barth-Institut.

Kühn, M., \& Hadorn, P. (2004). Pflanzliche Makro- und Mikroreste aus Dung von Wiederkäuern. In S. Jacomet, U. Leuzinger, \& J. Schibler (Eds.), Die jungsteinzeitliche Seeufersiedlung Arbon - Bleiche 3. Umwelt und Wirtschaft. Archäologie im Thurgau, 12, 327-350.

Kuper, R., \& Kröpelin, S. (2006). Climate-controlled Holocene occupation in the Sahara: Motor of Africa's evolution. Science, 313, 336-346.

Kweakason, A. P. (2008). Microwear analysis of some artefacts from archaeological horizon 5. In P. M. Vermeersch (Ed.), A Holocene prehistoric sequence in the Egyptian Red Sea area: The Tree Shelter (pp. 63-72). Leuven: Leuven University Press.

Lesur, J., Briois, F., Midant-Reynes, B., \& Wuttman, M. (2011). Domesticates and wild game in the Egyptian Western Desert at the end of the 5th millennium BC: The fauna from Ks 43, Kharga Oasis. In H. Jousse \& J. Lesur (Eds.), People and animals in Holocene Africa: Recent advances in archaeozoology (pp. 59-74). Frankfurt-am-Main: Africa Magna Verlag.

Linseele, V., Marinova, E., Van Neer, W., \& Vermeersch, P. M. (2010). Sites with Holocene dung deposits in the Eastern Desert of Egypt: Visited by herders? Journal of Arid Environments, 74, 818-828.

Linseele, V., \& Van Neer, W. (2008). Faunal remains from the Tree Shelter site. In P. M. Vermeersch (Ed.), A Holocene prehistoric sequence in the Egyptian Red Sea area: The Tree Shelter (pp. 63-72). Leuven: Leuven University Press. 
Marinova, E. (2008). Woody vegetation and its use during the Neolithic at the Tree Shelter. In P. M. Vermeersch (Ed.), A Holocene prehistoric sequence in the Egyptian Red Sea area: The Tree Shelter (pp. 73-78). Leuven: Leuven University Press.

Marinova, E., Linseele, V., \& Vermeersch, P. M. (2008). Holocene environment and subsistence patterns near the Tree Shelter, Red Sea Mountains, Egypt. Quaternary Research, 70, 392-397.

Marshall, M. H., Lamb, H. F., Huws, D., Davies, S. J., Bates, R., Bloemendal, J., Boyle, J., Leng, M. J., Umer, M., \& Bryant, C. (2011). Late Pleistocene and Holocene drought events at Lake Tana, the source of the Blue Nile. Global and Planetary Change, 78, 147-161.

McDonald, M. M. A. (1991). Origins of the Neolithic in the Nile Valley as seen from Dakhleh Oasis in the Egyptian Western Desert. Sahara, 4, 41-52.

McDonald, M. M. A. (1992). Neolithic of Sudanese tradition or Saharo-Sudanese Neolithic? The view from Dakhla Oasis, South Central Egypt. In J. Sterner \& N. David (Eds.), An African commitment: Papers in honour of P. L. Shinnie (pp. 51-70). Calgary: Calgary Press.

McDonald, M. M. A. (2003). The Early Holocene Masara A and Masara C cultural sub-units of Dakhleh Oasis, within a wider cultural setting. In G. E. Bowen \& C. A. Hope (Eds.), The Oasis Papers 3, Proceedings of the Third International Conference of the Dakhleh Oasis Project (pp. 43-70). Oxford: Oxbow Books.

McDonald, M. M. A. (2013). Whence the Neolithic of northeastern Africa? Evidence from the Central Western Desert of Egypt. In N. Shirai (Ed.), Neolithisation of northeastern Africa (pp. 175-192). Berlin: Ex Oriente.

Midant-Reynes, B. (2003). Aux origines de l'Egypte. Du Néolithique à l'émergence de l'Etat. Paris: Fayard.

Moeyersons, J., Vermeersch, P. M., Beeckman, H., \& Van Peer, P. (1999). Holocene environmental changes in the Gebel Umm Hammad, Eastern Desert, Egypt. Geomorphology, 26, 297-312.

Moeyersons, J., Vermeersch, P. M., \& Van Peer, P. (2002). Dry cave deposits and their palaeoenvironmental significance during the last $115 \mathrm{ka}$, Sodmein Cave, Red Sea Mountains, Egypt. Quaternary Science Reviews, 21, 837-851.

Moeyersons, J., Vermeersch, P. M., Van Peer, P., Van Neer, W., Beeckman, H., \& De Coninck, E. (1996). Sodmein cave site, Red Sea Mountains, Egypt: Development, stratigraphy and palaeoenvironment. In G. Pwiti \& R. Soper (Eds.), Aspects of African archaeology (pp. 53-62). Harare: University of Zimbabwe Publications.

Montenat, C. (1986). Un aperçu des industries préhistoriques du golfe de Suez et du littoral Egyptien de la Mer Rouge. Bulletin de l'Institut français d'Archéologie Orientale, 86, 239-255.

Muigai, A. T., \& Hanotte, O. (2013). The origin of African sheep: Archaeological and genetic perspectives. African Archaeological Review, 30, 39-50.

Osborn, D. J., \& Helmy, I. (1980). The contemporary land mammals of Egypt (including Sinai). Chicago: Fieldiana Zoology, New Series 5.

Pöllath, N. (2010). Prähistorische und rezente Fauneninventare vom Abu-Muharik-Plateau. In K. Kindermann (Ed.), Djara - Zur mittelholozänen Besiedlungsgeschichte zwischen Niltal und Oasen (Abu-MuharikPlateau, Ägypten), Teil 2 (pp. 836-858). Köln: Heinrich-Barth-Institut.

Prickett, M. (1979). Quseir regional survey. In D. S. Whitcomb \& J. H. Johnson (Eds.), Quseir Al-Qadim 1978 preliminary report (pp. 257-349). Cairo: American Research Center in Egypt.

Riemer, H., Kindermann, K., \& Eickelkamp, S. (2004). Dating and production technique of Ounan points in the Eastern Sahara. New archaeological evidence from Abu Tartur, Western Desert of Egypt. Nyame Akuma, 61, 10-16.

Riemer, H., \& Kindermann, K. (2007). Contacts between the Oasis and the Nile: A résumé of the Abu Muhariq Plateau Survey 1995-2002. In B. Midant-Reynes \& Y. Tristant (Eds.), Egypt at its origins 2. Orientalia Lovaniensia Analecta 172 (pp. 610-633). Leuven: Peeters Publishers.

Riemer, H., \& Schönfeld, P. (2010). The prehistoric pottery of the Abu Muhariq Plateau. In K. Kindermann (Ed.), Djara, Zu mittelholozänen Besiedlungsgeschichte zwischen Niltal and Oasen (Abu-MuharikPlateau, Ägyten) (pp. 715-766). Köln: Heinrich-Barth-Institut.

Schild, R., Chiemewska, M., \& Więckowska, H. (1968). The Arkinian and Shamarkian industries. In F. Wendorf (Ed.), The prehistory of Nubia (pp. 651-767). Dallas: Southern Methodist University.

Schild, R., \& Wendorf, F. (2013). Early and middle Holcoene paleoclimates in the southwestern desert of Egypt - the world before unification. Studia Quaternaria, 30, 125-133.

Shirai, N. (2010). The archaeology of the first farmer-herders in Egypt: New insights into the Fayum Epipalaeolithic and Neolithic. Leiden: Leiden University Press.

Thanheiser, U. (2011). Island of the blessed: 8000 years of plant exploitation in the Dakhleh Oasis, Egypt. In G. Fahmy, S. Kahlheber, \& A. C. D'Andrea (Eds.), Windows on the African past. Current approaches to African archaeobotany (pp. 79-90). Frankfurt-am-Main: Africa Magna Verlag. 
Thomas, E. R., Wolff, E. W., Mulvaney, R., Steffensen, J. P., Johnsen, S. J., Arrowsmith, C., White, J. W. C., Vaughn, B., \& Popp, T. (2007). The 8.2 ka event from Greenland ice cores. Quaternary Science Reviews, $26,70-81$.

Tristant, Y. (2010). Le desert oriental durant la préhistoire. Bref aperçu des travaux récents menés dans le Wâdî’Araba. Archéo-Nil, 20, 51-61.

Vermeersch, P. M. (1978). Elkab II. L’Elkabien, Epipaléolithique de la Vallée du Nil Egyptien. Leuven: Universitaire Pers Leuven.

Vermeersch, P. M. (2008). A Holocene prehistoric sequence in the Egyptian Red Sea area: The Tree Shelter. Leuven: Leuven University Press.

Vermeersch, P. M. (2012). Contributions to the prehistory of the Eastern Desert, Egypt. In H. Barnard \& K. Duistermaat (Eds.), The history of the peoples of the Eastern Desert (pp. 24-41). Los Angeles: Cotsen Institute of Archaeology at UCLA.

Vermeersch, P. M., Van Peer, P., Moeyersons, J., \& Van Neer, W. (1994). Sodmein cave site, Red mountains (Egypt). Sahara, 6, 32-40.

Vermeersch, P. M., Van Peer, P., Moeyersons, J., \& Van Neer, W. (1996). Neolithic occupation of the Sodmein area, Red Sea Mountains, Egypt. In G. Pwiti \& R. Soper (Eds.), Aspects of African archaeology (pp. 411420). Harare: University of Zimbabwe Publications.

Vermeersch, P. M., Van Peer, P., Rots, V., \& Paulussen, R. (2005a). A survey of the Bili-cave and its surroundings in the Red Sea mountains, El Gouna, Egypt. Journal of African Archaeology, 3, 267-276.

Vermeersch, P. M., Van Peer, P., Rots, V., Van Kerckhoven, L., \& Van Neer, W. (2005b). The middle Holocene shell mound of El Gouna on the Red Sea (Egypt). Journal of Field Archaeology, 30, 435-442.

Vermeersch, P. M., Van Peer, P., Van Neer, W., Rots, V., \& Ponzetta, L. (2007). A Palaeolithic site at Wadi Bili in the Red Sea mountains, Egypt. In N. Bicho (Ed.), From the Mediterranean basin to the Portuguese Atlantic shore: Papers in honor of Anthony Marks (pp. 69-81). Faro: Universidade do Algarve.

Vigne, J.-D., \& Helmer, D. (2007). Was milk a "secondary product" in the Old World Neolithisation process? Its role in the domestication of cattle, sheep and goats. Anthropozoologica, 42, 9-40.

Von Maydell, H. J. (1986). Trees and shrubs of the Sahel -their characteristics and uses. Eschborn: GTZ $6 \mathrm{MBH}$.

Wasylikowa, K. (2001). Site E-75-6: Vegetation and subsistence of the Early Neolithic at Nabta Playa, Egypt, reconstructed from charred plant remains. In F. Wendorf, R. Schild, \& Associates (Eds.), Holocene settlement of the Egyptian Sahara (pp. 544-591). New York: Kluwer.

Wendorf, F., Schild, R., \& Associates. (2001). Holocene settlement of the Egyptian Sahara. New York: Kluwer.

Wendrich, W., Taylor, R. E., \& Southon, J. (2010). Dating stratified settlement sites at Kom K and Kom W: Fifth millennium BCE radiocarbon ages for the Fayum Neolithic. Nucl Instrum Methods Phys Res B, 268, 999-1002.

Wilkinson, T., Butzer, K. W., Huyge, D., Hendrickx, S., Kendall, T., \& Shaw, I. (2004). Review feature: A review of Genesis of the pharaohs: Dramatic new discoveries that rewrite the origins of ancient Egypt, by T. Wilkinson. London: Thames \& Hudson, 2002. Cambridge Archaeological Journal, 14, 113-135. 